\title{
Prospects for Observing and Localizing Gravitational-Wave Transients with Advanced LIGO and Advanced Virgo
}

\author{
Abbott, B. P. et al. \\ The LIGO Scientific Collaboration and the Virgo Collaboration \\ (The full author list and affiliations are given at the end of paper.) \\ email: lsc-spokesperson@ligo.org, virgo-spokesperson@ego-gw.it
}

Accepted: 22 January 2016

Published: 8 February 2016

\begin{abstract}
We present a possible observing scenario for the Advanced LIGO and Advanced Virgo gravitational-wave detectors over the next decade, with the intention of providing information to the astronomy community to facilitate planning for multi-messenger astronomy with gravitational waves. We determine the expected sensitivity of the network to transient gravitational-wave signals, and study the capability of the network to determine the sky location of the source. We report our findings for gravitational-wave transients, with particular focus on gravitational-wave signals from the inspiral of binary neutron-star systems, which are considered the most promising for multi-messenger astronomy. The ability to localize the sources of the detected signals depends on the geographical distribution of the detectors and their relative sensitivity, and $90 \%$ credible regions can be as large as thousands of square degrees when only two sensitive detectors are operational. Determining the sky position of a significant fraction of detected signals to areas of $5 \mathrm{deg}^{2}$ to $20 \mathrm{deg}^{2}$ will require at least three detectors of sensitivity within a factor of $\sim 2$ of each other and with a broad frequency bandwidth. Should the third LIGO detector be relocated to India as expected, a significant fraction of gravitational-wave signals will be localized to a few square degrees by gravitational-wave observations alone.
\end{abstract}

Keywords: Gravitational waves, Gravitational-wave detectors, Electromagnetic counterparts, Data analysis

(c) The Author(s). This article is distributed under a Creative Commons Attribution 4.0 International License. http://creativecommons.org/licenses/by/4.0/ 


\section{Imprint / Terms of Use}

Living Reviews in Relativity is a peer-reviewed open access journal published by the Springer International Publishing AG, Gewerbestrasse 11, 6330 Cham, Switzerland. ISSN 1433-8351.

This article is distributed under the terms of the Creative Commons Attribution 4.0 International License (http://creativecommons.org/licenses/by/4.0/), which permits unrestricted use, distribution, and reproduction in any medium, provided you give appropriate credit to the original author(s) and the source, provide a link to the Creative Commons license, and indicate if changes were made. Figures that have been previously published elsewhere may not be reproduced without consent of the original copyright holders.

Abbott, B. P. et al.,

"Prospects for Observing and Localizing Gravitational-Wave Transients with Advanced LIGO and Advanced Virgo",

Living Rev. Relativity, 19, (2016), 1.

DOI 10.1007/lrr-2016-1.

\section{Article Revisions}

Living Reviews supports two ways of keeping its articles up-to-date:

Fast-track revision. A fast-track revision provides the author with the opportunity to add short notices of current research results, trends and developments, or important publications to the article. A fast-track revision is refereed by the responsible subject editor. If an article has undergone a fast-track revision, a summary of changes will be listed here.

Major update. A major update will include substantial changes and additions and is subject to full external refereeing. It is published with a new publication number.

For detailed documentation of an article's evolution, please refer to the history document of the article's online version at http://dx.doi.org/10.1007//rr-2016-1. 


\section{Contents}

1 Introduction $\quad 5$

2 Commissioning and Observing Phases 6

2.1 Commissioning and observing roadmap . . . . . . . . . . . . . . . 6

2.2 Envisioned observing schedule . . . . . . . . . . . . . . . . . 8

3 Searches for Gravitational-Wave Transients $\quad 9$

3.1 Detection and false alarm rates . . . . . . . . . . . . . . . . . . . 10

3.2 Sky localization . . . . . . . . . . . . . . . . . . . . 11

3.2.1 Localization of binary neutron stars . . . . . . . . . . . . . . . 13

3.2.2 Localization of bursts . . . . . . . . . . . . . . . . . . 14

4 Observing Scenario $\quad 18$

$4.12015-2016$ run $(\mathrm{O} 1):$ aLIGO $40-80 \mathrm{Mpc} \ldots \ldots \ldots \ldots$

4.22016 -2017 run (O2): aLIGO $80-120$ Mpc, AdV 20-60 Mpc . . . . . . . . . . 19

$4.32017-2018$ run (O3): aLIGO $120-170 \mathrm{Mpc}, \mathrm{AdV} 60-85 \mathrm{Mpc} \ldots \ldots . . . . . .20$

$4.42019+$ runs: aLIGO $200 \mathrm{Mpc}, \mathrm{AdV} 65-130 \mathrm{Mpc} \ldots \ldots \ldots . \ldots . . \ldots 20$

$4.52022+$ runs: aLIGO (including India) $200 \mathrm{Mpc}$, AdV $130 \mathrm{Mpc}$. . . . . . . . . 20

5 Conclusions $r$

A Changes Between Versions $\quad 25$

A.1 Updates to detector commissioning . . . . . . . . . . . . . . . . . . . 25

A.2 Updates to sky localization . . . . . . . . . . . . . . . . . . . . . . . . 25

$\begin{array}{lr}\text { References } & 26\end{array}$

\section{List of Tables}

1 Summary of a plausible observing schedule, expected sensitivities, and source localization with the advanced LIGO and Virgo detectors. 



\section{Introduction}

Advanced LIGO (aLIGO) [61, 9] and Advanced Virgo (AdV) [24, 23, 25] are kilometer-scale gravitational-wave $(\mathrm{GW})$ detectors that are expected to yield direct observations of GWs. In this article we describe the currently projected schedule, sensitivity, and sky-localization accuracy for the GW-detector network. We discuss the proposed sequence of observing runs (designated O1, O2, O3, etc.) and the prospects for multi-messenger astronomy.

The purpose of this article is to provide information to the astronomy community to assist in the formulation of plans for the upcoming era of GW observations. In particular, we intend this article to provide the information required for assessing the features of programs for joint observation of GW events using electromagnetic, neutrino, or other facilities.

The full science of aLIGO and AdV is broad [8], and is not covered in this article. We concentrate solely on candidate GW transient signals. We place particular emphasis on the coalescence of binary neutron-star (BNS) systems, which are the GW source for which electromagnetic follow-up seems most promising. For more general introductory articles on GW generation, detection and astrophysics, we point readers to [33, 87, 94].

Although our collaborations have amassed a great deal of experience with GW detectors and analysis, it is still difficult to make predictions for both improvements in search methods and for the rate of progress for detectors which are not yet fully installed or operational. The scenarios of LIGO and Virgo detector sensitivity evolution and observing times given here represent our best estimates as of January 2016. They should not be considered as fixed or firm commitments.

As the detectors' construction and commissioning progress, we intend to release updated versions of this article. This is the second version of the article, written to coincide with the first observing run (O1) of the advanced-detector era. Changes with respect to the first version [4] are given in Appendix A. Progress has been made in the commissioning of the detectors, and the plausible observing scenarios are largely the same; the predicted sky-localization accuracies have been updated following improvements in parameter estimation. 


\section{Commissioning and Observing Phases}

We divide the development of the aLIGO and AdV observatories into three components:

Construction includes the installation and testing of the detectors. This phase ends with acceptance of the detectors. Acceptance means that the interferometers can lock for periods of hours: light is resonant in the arms of the interferometer with no guaranteed $G W$ sensitivity. Construction incorporates several short engineering runs with no astrophysical output as the detectors progress towards acceptance. The aLIGO construction project ended (on time and on budget) in March 2015. The acceptance of AdV is expected in the first part of 2016.

Commissioning takes the detectors from their configuration at acceptance through progressively better sensitivity to the design advanced-generation detector sensitivity. Engineering runs in the commissioning phase allow us to understand our detectors and analyses in an observational mode; these are not intended to produce astrophysical results, but that does not preclude the possibility of this happening. Rather than proceeding directly to design sensitivity before making astrophysical observations, commissioning is interleaved with observing runs of progressively better sensitivity.

Observing runs begin when the detectors have reached (and can stably maintain) a significantly improved sensitivity compared with previous operation. It is expected that observing runs will produce astrophysical results, including upper limits on the rate of sources and possibly the first detections of GWs. During this phase, exchange of GW candidates with partners outside the LIGO Scientific Collaboration (LSC) and the Virgo Collaboration will be governed by memoranda of understanding (MOUs) [17, 2]. After the first four detections, we expect free exchange of GW event candidates with the astronomical community and the maturation of GW astronomy.

The progress in sensitivity as a function of time will affect the duration of the runs that we plan at any stage, as we strive to minimize the time to successful GW observations. Commissioning is a complex process which involves both scheduled improvements to the detectors and tackling unexpected new problems. While our experience makes us cautiously optimistic regarding the schedule for the advanced detectors, we are targeting an order of magnitude improvement in sensitivity relative to the previous generation of detectors over a wider frequency band. Consequently, it is not possible to make concrete predictions for sensitivity or duty cycle as a function of time. We can, however, use our experience as a guide to plausible scenarios for the detector operational states that will allow us to reach the desired sensitivity. Unexpected problems could slow down the commissioning, but there is also the possibility that progress may happen faster than predicted here. As the detectors begin to be commissioned, information on the cost in time and benefit in sensitivity will become more apparent and drive the schedule of runs. More information on event rates, including the first detection, could also change the schedule and duration of runs.

In Section 2.1 we present the commissioning plans for the aLIGO and AdV detectors. A summary of expected observing runs is in Section 2.2.

\subsection{Commissioning and observing roadmap}

The anticipated strain sensitivity evolution for aLIGO and AdV is shown in Figure 1. A standard figure of merit for the sensitivity of an interferometer is the BNS range $R_{\mathrm{BNS}}$ : the volume- and orientation-averaged distance at which a compact binary coalescence consisting of two $1.4 M_{\odot}$ neutron stars gives a matched filter signal-to-noise ratio (SNR) of 8 in a single detector [58]. ${ }^{1}$ The

\footnotetext{
1 Another often quoted number is the BNS horizon - the distance at which an optimally oriented and located BNS system would be observed with an SNR of 8 . The horizon is a factor of 2.26 larger than the range [58, 13, 20].
} 
BNS ranges for the various stages of aLIGO and AdV expected evolution are also provided in Figure 1.
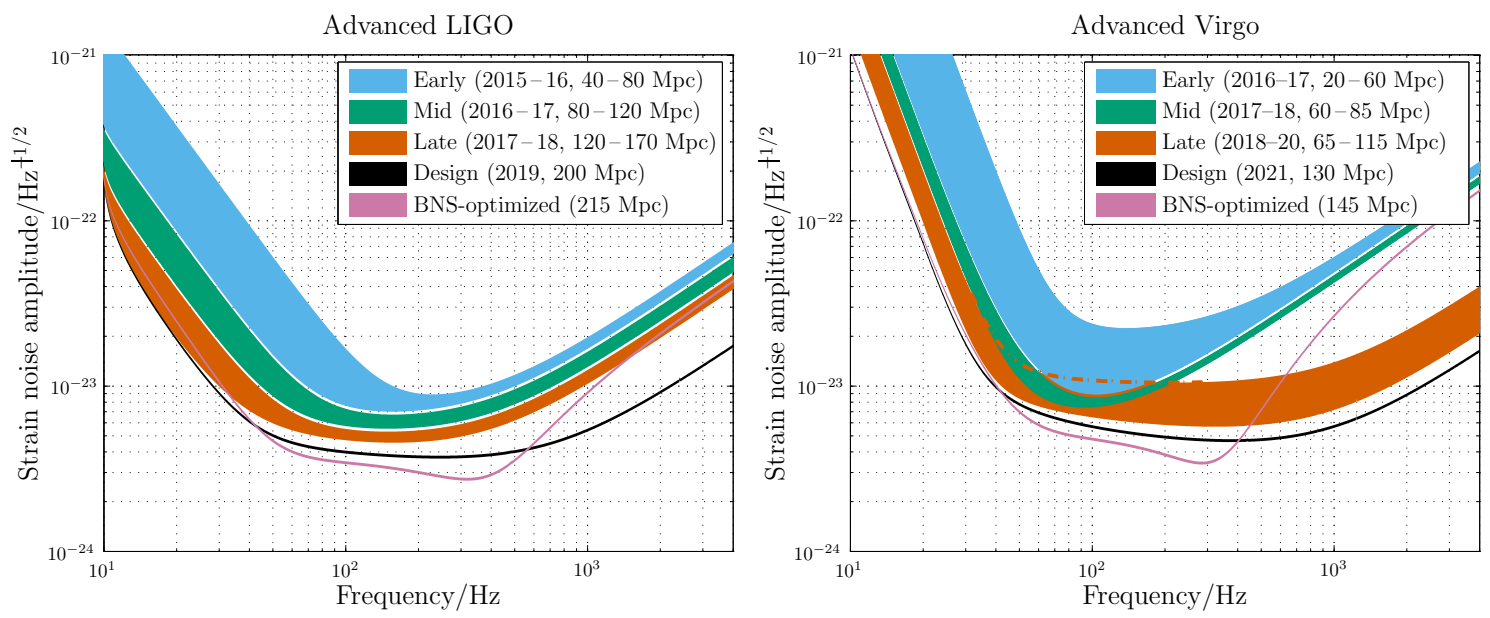

Figure 1: aLIGO (left) and AdV (right) target strain sensitivity as a function of frequency. The binary neutron-star (BNS) range, the average distance to which these signals could be detected, is given in megaparsec. Current notions of the progression of sensitivity are given for early, mid and late commissioning phases, as well as the final design sensitivity target and the BNS-optimized sensitivity. While both dates and sensitivity curves are subject to change, the overall progression represents our best current estimates.

The commissioning of aLIGO is well under way. The original plan called for three identical 4-km interferometers, two at Hanford (H1 and H2) and one at Livingston (L1). In 2011, the LIGO Lab and IndIGO consortium in India proposed installing one of the aLIGO Hanford detectors (H2) at a new observatory in India (LIGO-India) [64]. As of early 2015, LIGO Laboratory has placed the $\mathrm{H} 2$ interferometer in long-term storage for possible use in India. Funding for the Indian portion of LIGO-India is in the final stages of consideration by the Indian government.

Advanced LIGO detectors began taking sensitive data in August 2015 in preparation for the first observing run. O1 formally began 18 September 2015 and ended 12 January 2016. It involved the $\mathrm{H} 1$ and L1 detectors; the detectors were not at full design sensitivity. We aimed for a BNS range of 40-80 Mpc for both instruments (see Figure 1), and both instruments were running with a 60-80 Mpc range. Subsequent observing runs will have increasing duration and sensitivity. We aim for a BNS range of $80-170 \mathrm{Mpc}$ over 2016-2018, with observing runs of several months. Assuming that no unexpected obstacles are encountered, the aLIGO detectors are expected to achieve a $200 \mathrm{Mpc}$ BNS range circa 2019. After the first observing runs, circa 2020, it might be desirable to optimize the detector sensitivity for a specific class of astrophysical signals, such as BNSs. The BNS range may then become $215 \mathrm{Mpc}$. The sensitivity for each of these stages is shown in Figure 1.

As a consequence of the planning for the installation of one of the LIGO detectors in India, the installation of the $\mathrm{H} 2$ detector has been deferred. This detector will be reconfigured to be identical to H1 and L1 and will be installed in India once the LIGO-India Observatory is complete. The final schedule will be adopted once final funding approvals are granted. If project approval comes soon, site development could start in 2016, with installation of the detector beginning in 2020. Following this scenario, the first observing runs could come circa 2022, and design sensitivity at the same level as the H1 and L1 detectors is anticipated for no earlier than 2024.

The time-line for the AdV interferometer (V1) [23] is still being defined, but it is anticipated that in $2016 \mathrm{AdV}$ will join the aLIGO detectors in their second observing run (O2). Following an early step with sensitivity corresponding to a BNS range of 20-60 Mpc, commissioning is expected 
to bring AdV to a $60-85 \mathrm{Mpc}$ in $2017-2018$. A configuration upgrade at this point will allow the range to increase to approximately $65-115 \mathrm{Mpc}$ in $2018-2020$. The final design sensitivity, with a BNS range of $130 \mathrm{Mpc}$, is anticipated circa 2021. The corresponding BNS-optimized range would be $145 \mathrm{Mpc}$. The sensitivity curves for the various AdV configurations are shown in Figure 1.

The GEO 600 [76] detector will likely be operational in the early to middle phase of the AdV and aLIGO observing runs, i.e. $2015-2017$. The sensitivity that potentially can be achieved by GEO in this time-frame is similar to the $\mathrm{AdV}$ sensitivity of the early and mid scenarios at frequencies around $1 \mathrm{kHz}$ and above. GEO could therefore contribute to the detection and localization of high-frequency transients in this period. However, in the $\sim 100 \mathrm{~Hz}$ region most important for BNS signals, GEO will be at least 10 times less sensitive than the early AdV and aLIGO detectors, and will not contribute significantly.

Japan has begun the construction of an advanced detector, KAGRA [100, 28]. KAGRA is designed to have a BNS range comparable to AdV at final sensitivity. We do not consider KAGRA in this article, but the addition of KAGRA to the worldwide GW-detector network will improve both sky coverage and localization capabilities beyond those envisioned here [96].

Finally, further upgrades to the LIGO and Virgo detectors, within their existing facilities (e.g., $[63,78,11]$ ) as well as future underground detectors (for example, the Einstein Telescope [93]) are envisioned in the future. These affect both the rates of observed signals as well as the localizations of these events, but this lies beyond the scope of this paper.

\subsection{Envisioned observing schedule}

Keeping in mind the important caveats about commissioning affecting the scheduling and length of observing runs, the following is a plausible scenario for the operation of the LIGO-Virgo network over the next decade:

2015 - 2016 (O1) A four-month run (beginning 18 September 2015 and ending 12 January 2016) with the two-detector H1L1 network at early aLIGO sensitivity (40-80 Mpc BNS range).

2016-2017 (O2) A six-month run with H1L1 at 80-120 Mpc and V1 at 20-60 Mpc.

2017-2018 (O3) A nine-month run with H1L1 at 120-170 Mpc and V1 at 60-85 Mpc.

2019+ Three-detector network with H1L1 at full sensitivity of $200 \mathrm{Mpc}$ and V1 at 65 - $115 \mathrm{Mpc}$.

2022+ H1L1V1 network at full sensitivity (aLIGO at $200 \mathrm{Mpc}$, AdV at $130 \mathrm{Mpc}$ ), with other detectors potentially joining the network. Including a fourth detector improves sky localization $[72,109,79,91]$, so as an illustration we consider adding LIGO-India to the network. 2022 is the earliest time we imagine LIGO-India could be operational, and it would take several more years for it to achieve full sensitivity.

This time-line is summarized in Figure 2. The observational implications of this scenario are discussed in Section 4. 


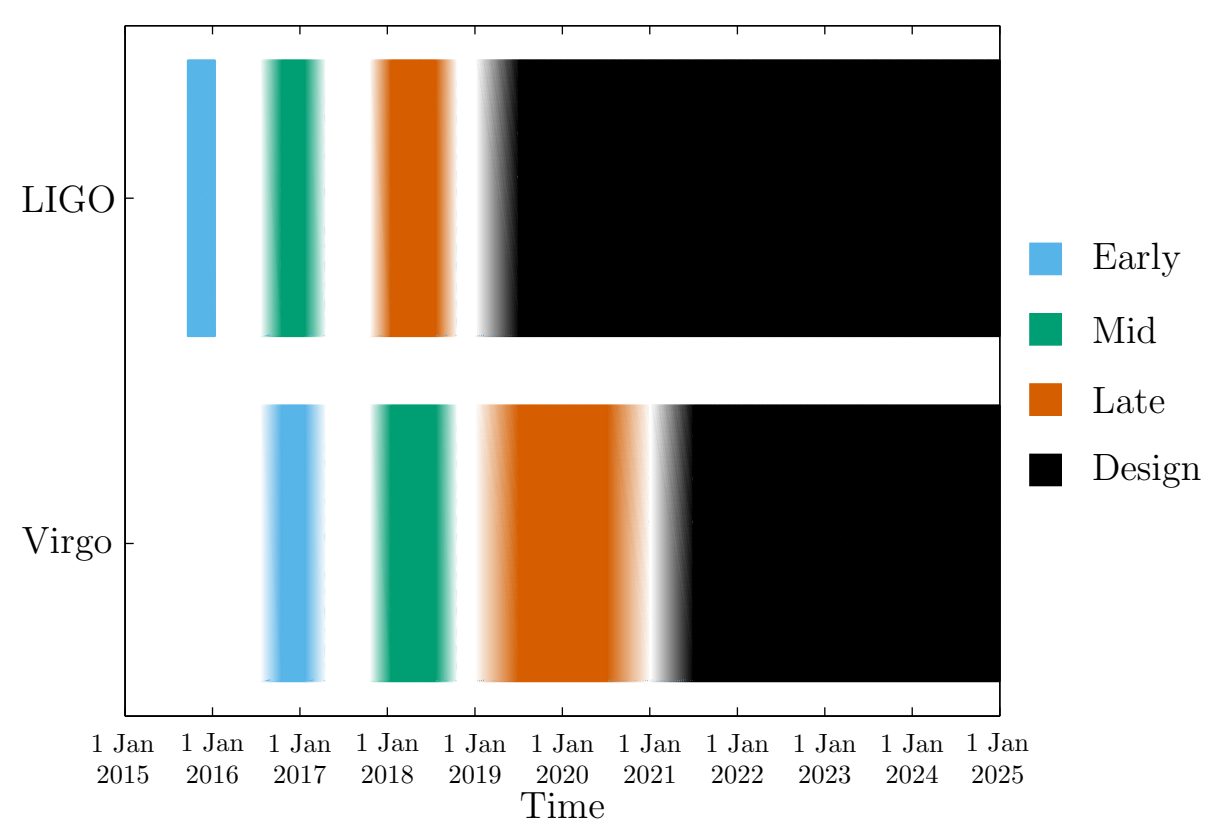

Figure 2: The planned sensitivity evolution and observing runs of the aLIGO and AdV detectors over the coming years. The colored bars show the observing runs, with the expected sensitivities given by the data in Figure 1. There is significant uncertainty in the start and end times of the observing runs, especially for those further in the future, and these could move forward or backwards by a few months relative to what is shown above. The plan is summarised in Section 2.2.

\section{Searches for Gravitational-Wave Transients}

Data from GW detectors are searched for many types of possible signals [8]. Here we focus on signals from compact binary coalescences (CBCs), including BNS systems, and on generic transient or burst signals. See $[19,18,14]$ for observational results from LIGO and Virgo for such systems.

The rate of BNS coalescences is uncertain [54]. For this work we adopt the estimates of [13], which predicts the rate to lie between $10^{-8}-10^{-5} \mathrm{Mpc}^{-3} \mathrm{yr}^{-1}$, with a most plausible value of $10^{-6} \mathrm{Mpc}^{-3} \mathrm{yr}^{-1}$; this corresponds to $0.4-400$ signals above an SNR of 8 per year of observation for a single aLIGO detector at final sensitivity, and a best estimate of 40 BNS signals per year [13]. Rate estimation remains an active area of research (e.g., [69, 50, 49, 47]), and will be informed by the number of detections (or lack thereof) in observing runs.

While the intrinsic rates of neutron star-black hole (NS-BH) and binary black hole $(\mathrm{BBH})$ mergers are expected to be a factor of tens or hundreds lower than the BNS rate, the distance to which they can be observed is a factor of two to five larger. Consequently, the predicted observable rates are similar $[13,92]$. Expected rates for other transient sources are lower and/or less well constrained.

The gravitational waveform from a BNS coalescence is well modeled and matched filtering can be used to search for signals and measure the system parameters [74, 35, 34, 90]. For systems containing black holes, or in which the component spin is significant, uncertainties in the waveform model can reduce the sensitivity of the search [81, 62, 45, 103, 85, 95, 68]. Searches for bursts make few assumptions on the signal morphology, using time-frequency decompositions to identify statistically significant excess-power transients in the data. Burst searches generally perform best for short-duration signals $(\lesssim 1 \mathrm{~s})$, although search development remains an area of active research (e.g., $[71,102,40,106,26,104,43,105,66])$; their astrophysical targets include core-collapse supernovae, 
magnetar flares, BBH coalescences, cosmic string cusps, and, possibly, as-yet-unknown systems.

In the era of advanced detectors, the LSC and Virgo will search in near real-time for CBC and burst signals for the purpose of rapidly identifying event candidates. A prompt notice of a potential GW transient by LIGO-Virgo might enable follow-up observations in the electromagnetic spectrum. A first follow-up program including low-latency analysis, event candidate selection, position reconstruction and the sending of alerts to several observing partners (optical, X-ray, and radio) was implemented and exercised during the 2009-2010 LIGO-Virgo science run [16, 15, 53]. Latencies of less than 1 hour were achieved and we expect to improve this in the advanced-detector era. Increased detection confidence, improved sky localization, and identification of host galaxy and redshift are just some of the benefits of joint GW-electromagnetic observations. With this in mind, we focus on two points of particular relevance for follow-up of GW events: the source localization afforded by a GW network as well as the relationship between signal significance, or false alarm rate (FAR), and source localization.

\subsection{Detection and false alarm rates}

The rate of false alarm triggers above a given SNR depends critically upon the data quality of the advanced detectors; non-stationary transients or glitches $[1,10]$ produce an elevated background of loud triggers. For low-mass binary coalescence searches, the waveforms are well modeled, and signal consistency tests reduce the background significantly [27, 38, 107]. For burst sources which are not well modeled, or which spend only a short time in the detectors' sensitive band, it is more difficult to distinguish between the signal and a glitch, and so a reduction of the FAR comes at a higher cost in terms of reduced detection efficiency.
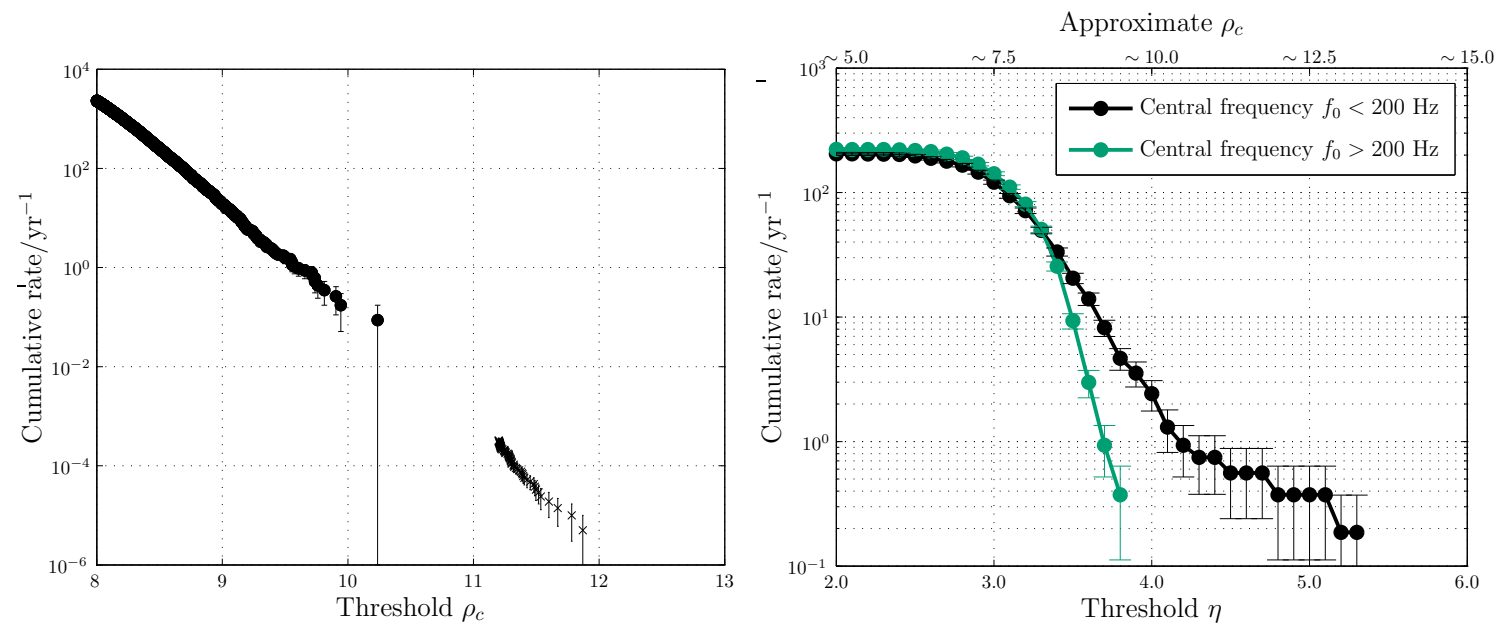

Figure 3: False alarm rate versus detection statistic for compact binary coalescence (CBC) and burst searches on 2009-2010 LIGO-Virgo data. Left: Cumulative rate of background events for a subset of the $\mathrm{CBC}$ search parameter space, as a function of the threshold ranking statistic $\rho_{c}$ [19]. Different methods were used to estimate the background for rate for high and low $\rho_{c}$, which is why there is an apparent gap in the data points. The background for the full search was approximately a factor of six higher. Right: Cumulative rate of background events for the burst search, as a function of the coherent network amplitude $\eta$ [14]. For ease of comparison, we have also plotted the approximate equivalent $\rho_{c}$ for the burst search (an exact identification is not possible as the search methods differ). The burst events are divided into two sets based on their central frequency.

Figure 3 shows the noise background as a function of detection statistic for the low-mass binary 
coalescence and burst searches with the 2009-2010 LIGO-Virgo data [19, 14]. ${ }^{2}$ For binary mergers, the background rate decreases by a factor of $\sim 100$ for every unit increase in combined SNR $\rho_{c}$. Here $\rho_{c}$ is a combined, re-weighted SNR [29, 19]. The re-weighting is designed to reduce the SNR of glitches while leaving signals largely unaffected. Consequently, for a signal, $\rho_{c}$ is essentially the root-sum-square of the SNRs in the individual detectors. For bursts, we use the coherent network amplitude $\eta$, which measures the degree of correlation between the detectors [22, 86]. Glitches have little correlated energy and so give low values of $\eta$. Both $\rho_{c}$ and $\eta$ give an indication of the amplitude of the signal and can be used to rank events.

For CBC signals, we conservatively estimate that a $\rho_{c}$ threshold of 12 is required for a FAR below $\sim 10^{-2} \mathrm{yr}^{-1}$ in aLIGO-AdV. To arrive at this estimate, we begin with Figure 3 which indicates that an SNR of around 10.5 corresponds to a FAR of $10^{-2} \mathrm{yr}^{-1}$. However, this corresponds to only a subspace of the CBC parameter space and the background of the full search is a factor of six higher. Additionally, due to the improved low frequency sensitivity of the advanced detectors and the inclusion of templates for binaries with (aligned) component spins, at least ten times as many waveform templates are required to perform the search [84, 34, 88, 46]. The background increases approximately linearly with the number of templates required. Consequently, we expect a background around a factor of 100 higher than indicated by Figure 3, which leads us to quote a threshold of 12 for a FAR of $10^{-2} \mathrm{yr}^{-1}$ [31]. A combined SNR of 12 corresponds to a single-detector SNR of 8.5 in each of two detectors, or 7 in each of three detectors.

Instrumental disturbances in the data can have a greater effect on the burst search. At frequencies above $200 \mathrm{~Hz}$, the rate of background events falls off steeply as a function of amplitude. At lower frequencies, however, the data often exhibit a significant tail of loud background events that are not simply removed by multi-detector consistency tests. Although the advanced detectors are designed with many technical improvements, we anticipate that similar features will persist, particularly at low frequencies. Improvements to detection pipelines, which better distinguish between glitches and potential waveforms, can help eliminate these tails (e.g., [66]). For a given FAR, the detection threshold may need to be tuned for different frequency ranges; for the initial detectors, a threshold of $\eta \gtrsim 4.5-6$ (approximately equivalent to $\rho_{c} \gtrsim 12$ from Figure 3 ) was needed for a FAR of $1 / 8 \mathrm{yr}^{-1}[14]$. The unambiguous observation of an electromagnetic counterpart could increase the detection confidence.

\subsection{Sky localization}

Following the detection by the aLIGO-AdV network of a GW transient, determining the source's location is a question for parameter estimation. Typically, posterior probability distributions for the sky position are constructed following a Bayesian framework [110, 43, 98]. Information comes from the time of arrival, plus the phase and amplitude of the GW.

An intuitive understanding of localization can be gained by considering triangulation using the observed time delays between sites $[55,56]$. The effective single-site timing accuracy is approximately

$$
\sigma_{t}=\frac{1}{2 \pi \rho \sigma_{f}},
$$

where $\rho$ is the SNR in the given detector and $\sigma_{f}$ is the effective bandwidth of the signal in the detector, typically of order $100 \mathrm{~Hz}$. Thus a typical timing accuracy is on the order of $10^{-4} \mathrm{~s}$ (about 1/100 of the light travel time between sites). This sets the localization scale. The simple model of equation (1) ignores many other relevant issues such as information from the signal amplitudes across the detector network, uncertainty in the emitted gravitational waveform, instrumental calibration accuracies, and correlation of sky location with other binary parameters [55, 112, 111, 80, 109, 79, 99, 31, 98]. While many of these affect the measurement of the time of arrival in individual detectors, such

\footnotetext{
${ }^{2}$ Data from the fifth and sixth science runs (S5 and S6) of LIGO are publicly available from the LIGO Open Science Center losc.ligo.org [108].
} 
factors are largely common between two similar detectors, so the time difference between the two detectors is relatively uncorrelated with these nuisance parameters.

The triangulation approach underestimates how well a source can be localized, since it does not include all the relevant information. Its predictions can be improved by introducing the requirement of phase consistency between detectors [60]. Triangulation always performs poorly for a two-detector network, but, with the inclusion of phase coherence, can provide an estimate for the average performance of a three-detector network [31]. ${ }^{3}$

Source localization using only timing for a two-site network yields an annulus on the sky; see Figure 4. Additional information such as signal amplitude, spin, and precession effects resolve this to only parts of the annulus, but even then sources will only be localized to regions of hundreds to thousands of square degrees [99, 31]. An example of a two-detector BNS localization is shown in Figure 5. The posterior probability distribution is primarily distributed along a ring, but this ring is broken, such that there are clear maxima.

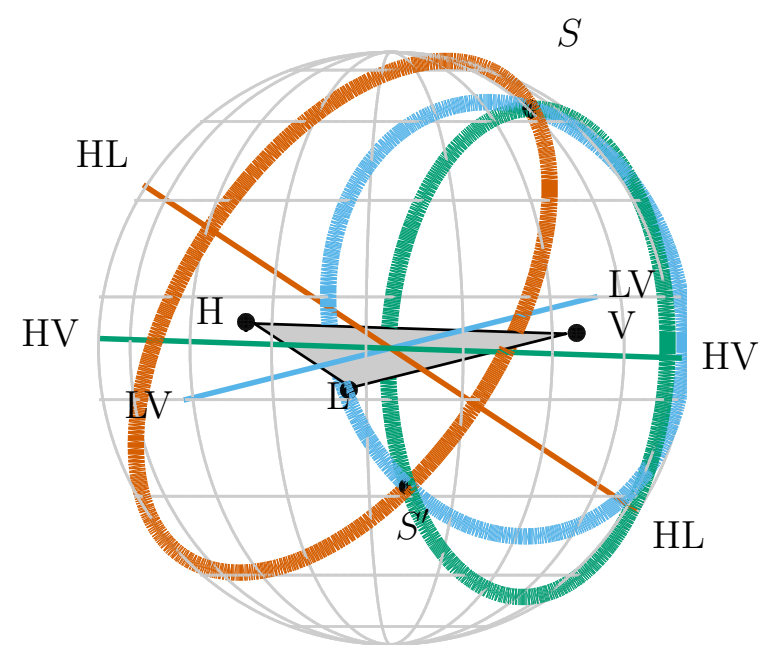

Figure 4: Source localization by triangulation for the aLIGO-AdV network. The locations of the three detectors are indicated by black dots, with LIGO Hanford labeled H; LIGO Livingston as L, and Virgo as $\mathrm{V}$. The locus of constant time delay (with associated timing uncertainty) between two detectors forms an annulus on the sky concentric about the baseline between the two sites (labeled by the two detectors). For three detectors, these annuli may intersect in two locations. One is centered on the true source direction $(S)$, while the other $\left(S^{\prime}\right)$ is its mirror image with respect to the geometrical plane passing through the three sites. For four or more detectors there is a unique intersection region of all of the annuli. Figure adapted from [41].

For three detectors, the time delays restrict the source to two sky regions which are mirror images with respect to the plane passing through the three sites. It is often possible to eliminate one of these regions by requiring consistent amplitudes in all detectors. For signals just above the detection threshold, this typically yields regions with areas of several tens to hundreds of square degrees. Additionally, for BNSs, it is often possible to obtain a reasonable estimate of the distance to the source $[109,31]$, which can be used to further aid electromagnetic observations [79, 32]. If there is significant difference in sensitivity between detectors, the source is less well localized and we may be left with the majority of the annulus on the sky determined by the two most sensitive detectors. With four or more detectors, timing information alone is sufficient to localize to a single sky region, and the additional baselines help to limit the region to under $10 \mathrm{deg}^{2}$ for some signals.

\footnotetext{
3 We do not intend to produce timing-only sky maps, but timing triangulation can be useful for order-of-magnitude estimates of sky-localization accuracy averaged across the population of signals.
} 

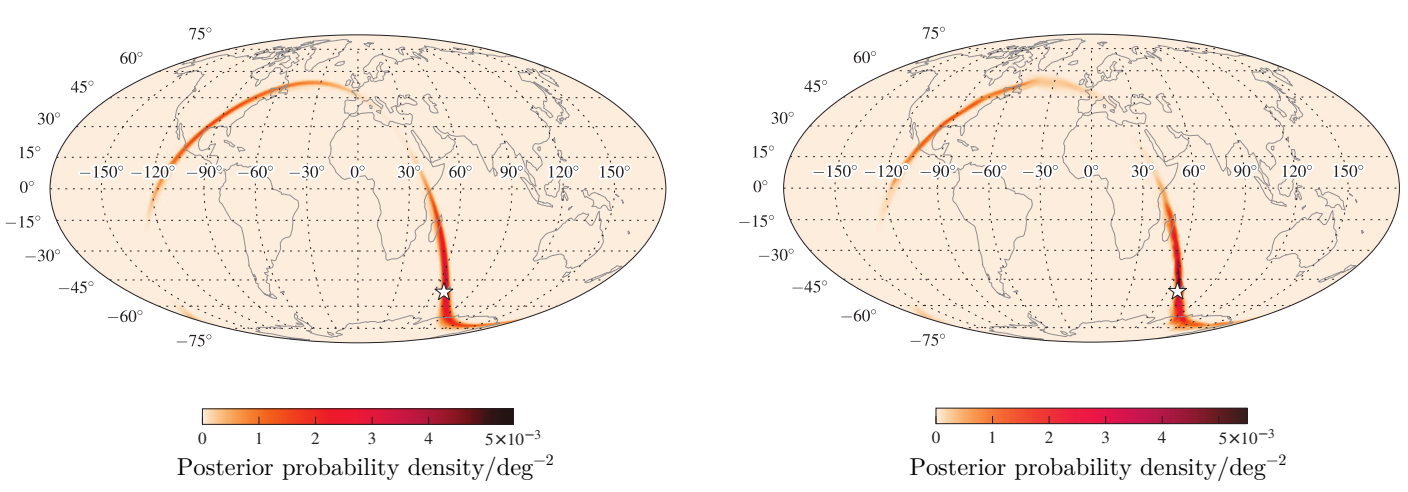

Figure 5: Posterior probability density for sky location for an example binary neutron-star coalescence observed with a two-detector network. Left: Map produced by the low-latency BAYESTAR code [99, 98]. Right: Map produced by the higher-latency (non-spinning) LALINFERENCE [110], which also produces posterior estimates for other parameters. These algorithms are discussed in Section 3.2.1. The star indicates the true source location. The event has a network signal-to-noise ratio of $\rho_{c}=13.2$ using a noise curve appropriate for the first aLIGO run (O1, see Section 4.1). The plot is a Mollweide projection in geographic coordinates. Image reproduced with permission from [31], copyright by APS; further mock sky maps for the first two observing runs can be found at www.ligo.org/scientists/first2years/ for binary neutron-star signals and www.ligo.org/scientists/burst-first2years/ for burst signals.

From Eq. (1), it follows that the linear size of the localization ellipse scales inversely with the SNR of the signal and the frequency bandwidth of the signal in the detector [31]. For GWs that sweep across the band of the detector, such as CBC signals, the effective bandwidth is $\sim 100 \mathrm{~Hz}$, determined by the most sensitive frequencies of the detector. For shorter transients the bandwidth $\sigma_{f}$ depends on the specific signal. For example, GWs emitted by various processes in core-collapse supernovae are anticipated to have relatively large bandwidths, between $150-500 \mathrm{~Hz}$ $[48,82,113,83]$, largely independent of detector configuration. By contrast, the sky localization region for narrowband burst signals may consist of multiple disconnected regions and exhibit fringing features; see for example $[72,16,51]$.

Some GW searches are triggered by electromagnetic observations, and in these cases localization information is known a priori. For example, in GW searches triggered by gamma-ray bursts $[18,7,6]$ the triggering space-based telescope provides the localization. The detection of a $\mathrm{GW}$ transient associated with a gamma-ray burst would provide strong evidence for the burst progenitor; for example, BNS mergers are considered the likely progenitors of most short gamma-ray bursts. It is therefore important to have high-energy telescopes operating during the advanced-detector era. Furthermore, the rapid identification of a GW counterpart to such a trigger could prompt further spectroscopic studies and longer, deeper follow-up in different wavelengths that may not always be done in response to gamma-ray bursts. This is particularly important for gamma-ray bursts with larger sky localization uncertainties, such as those reported by the Fermi GBM, which are not followed up as frequently as bursts reported by Swift.

Finally, all GW data are stored permanently, so that it is possible to perform retroactive analyses at any time.

\subsubsection{Localization of binary neutron stars}

Providing prompt localizations for GW signals helps to maximise the chance that electromagnetic observatories can catch a counterpart. Sky maps will be produced at several different latencies, with updates coming from more computationally expensive algorithms that refine our understanding of the source. For BNS signals, rapid sky localization is performed using BAYESTAR [98], a Bayesian 
parameter-estimation code that computes source location using output from the detection pipeline. It can produce sky maps (as in Figure 5) with latencies of only a few seconds. A similar approach to low-latency localization has been separately developed by [42], who find results consistent with BAYESTAR.

At higher latency, CBC parameter estimation is performed using the stochastic sampling algorithms of LALINFERENCE [110]. LALINFERENCE constructs posterior probability distributions for system parameters (not just location, but also mass, orientation, etc. [3]) by matching GW templates to the detector strain [44, 65]. Computing these waveforms is computationally expensive; this expense increases as the detectors' low-frequency sensitivity improves and waveforms must be computed down to lower frequencies. The quickest LALINFERENCE BNS follow-up is computed using waveforms that do not include the full effects of component spin [99, 31], sky locations can then be reported with latency of hours to a couple of days. Parameter estimation is then performed using more accurate waveform approximates (those that include the full effects of spin precession), which can take weeks or months [57]. Methods of reducing the computational cost are actively being investigated (e.g., [37, 89, 36]).

There is negligible difference between the low-latency BAYESTAR and the mid-latency nonspinning LALINFERENCE analyses if the BNS signal is loud enough to produce a trigger in all detectors: if there is not, LALINFERENCE currently gives a more precise localization, as it still uses information from the non-triggered detector [99, 98]. It is hoped to improve BAYESTAR localization in the future by including information about sub-threshold signals. Provided that BNSs are slowly spinning [77], there should be negligible difference between the mid-latency non-spinning LALINFERENCE and the high-latency fully spinning LALINFERENCE analyses [57].

Results from an astrophysically motivated population of BNS signals, assuming a detection threshold of a SNR of 12, are shown in Figure 6 [99, 31]. Sky localization is measured by the $90 \%$ credible region $\mathrm{CR}_{0.9}$, the smallest area enclosing $90 \%$ of the total posterior probability, and the searched area $A_{*}$, the area of the smallest credible region that encompasses the true position [97]: $\mathrm{CR}_{0.9}$ gives the area of the sky that must be covered to expect a $90 \%$ chance of including the source location, and $A_{*}$ gives the area that would be viewed before the true location using the given sky map. Results from both the low-latency BAYESTAR and mid-latency non-spinning LALINFERENCE analyses are shown. These are discussed further in Section 4.1 and Section 4.2. The two-detector localizations get slightly worse going from $\mathrm{O} 1$ to $\mathrm{O} 2$. This is because although the detectors improve in sensitivity at every frequency, with the assumed noise curves the BNS signal bandwidth is lower in O2 for a given SNR because of enhanced sensitivity at low frequencies [99]. Despite this, the overall sky-localization in $\mathrm{O} 2$ is better than in O1. As sky localization improves with the development of the detector network [96, 72, 109, 91], these results mark the baseline of performance for the advanced-detector era.

\subsubsection{Localization of bursts}

The lowest latency burst sky maps are produced as part of the COHERENT WAVEBurst (CWB) [71, 73] detection pipeline, earlier versions of which were used in prior burst searches [12, 14, 70]. Sky maps are produced using a constrained likelihood algorithm that coherently combines data from all the detectors; unlike other approaches, this is not fully Bayesian. The resulting sky maps currently show calibration issues. ${ }^{4}$ The confidence is over-estimated at low levels and under-estimated at high levels. However, the searched areas are consistent with those from other burst techniques, indicating that CWB maps can be used to guide observations. The CWB sky maps calculated with a latency of a few minutes; following detection, further parameter-estimation codes analyze the data.

\footnotetext{
4 We expect that on average $50 \%$ of sources should be found within a reported $50 \%$ confidence region, but this is not the case: between $65 \%$ and $85 \%$ of detected signals have been found within the $50 \%$ confidence regions, depending upon the detector network and signal morphology [51].
} 



Figure 6: Anticipated binary neutron-star sky localization during the first two observing runs (top: O1, see Section 4.1; bottom: O2, see Section 4.2). The plots show the cumulative fractions of events with sky-localization areas smaller than the abscissa value. Left: Sky area of $90 \%$ credible region $\mathrm{CR}_{0.9}^{\mathrm{BNS}}$, the (smallest) area enclosing $90 \%$ of the total posterior probability. Right: Searched area $A_{*}^{\mathrm{BNS}}$, the area of the smallest credible region containing the true position. Results are shown for the low-latency BAYESTAR [98] and higher-latency (non-spinning) LALINFEREnCE (LI) [110] codes. The O2 results are divided into those where two detectors (2-det) are operating in coincidence, and those where three detectors (3-det) are operating: assuming a duty cycle of $\sim 80 \%$ for each instrument, the two-detector network would be operating for $\sim 40 \%$ of the total time and the three-detector network $\sim 50 \%$ of the time. The shaded areas indicate the $68 \%$ confidence intervals on the cumulative distributions. A detection threshold of a signal-to-noise ratio of 12 is used and results are taken from [31, 99]. 
At higher latency, burst signals are analyzed by LALINFERENCEBURST (LIB), a stochastic sampling algorithm similar to the LALINFERENCE code used to reconstruct CBC signals [110], and BAYESWAVE, a reversible jump Markov-chain Monte Carlo algorithm that models both signals and glitches [43]. LIB uses a sine-Gaussian waveform (in place of the CBC templates used by LALINFERENCE), and can produce sky maps in a few hours. BAYESWAVE uses a variable number of sine-Gaussian wavelets to model a signal and glitches while also fitting for the noise spectrum using BAYEsLine [75]; it produces sky maps with a latency of a few days. Sky maps produced by LIB and BAYESWAVE should be similar, with performance depending upon the actual waveform.

A full study of burst localization in the first two years of the aLIGO-AdV network was completed in [51]. This study was conducted using CWB and LIB. Sky localization was quantified by the searched area. Using an approximate FAR threshold of $1 \mathrm{yr}^{-1}$, the median localization was shown to be $\sim 100-200 \mathrm{deg}^{2}$ for a two-detector network in 2015-2016 and $\sim 60-110 \mathrm{deg}^{2}$ for a three-detector network in 2016-2017, with the localization and relative performance of the algorithms depending upon the waveform morphology. Results for Gaussian, sine-Gaussian, broadband white-noise and $\mathrm{BBH}$ waveforms are shown in Figure 7 (for the two-detector O1 network and the three-detector O2 network, cf. Figure 6). The largest difference between the codes here is for the white-noise bursts; for a three-detector network, LIB achieves better localization than CWB for Gaussian and sine-Gaussian bursts. The variety of waveform morphologies reflect the range of waveforms that could be detected in a burst search [16]. 

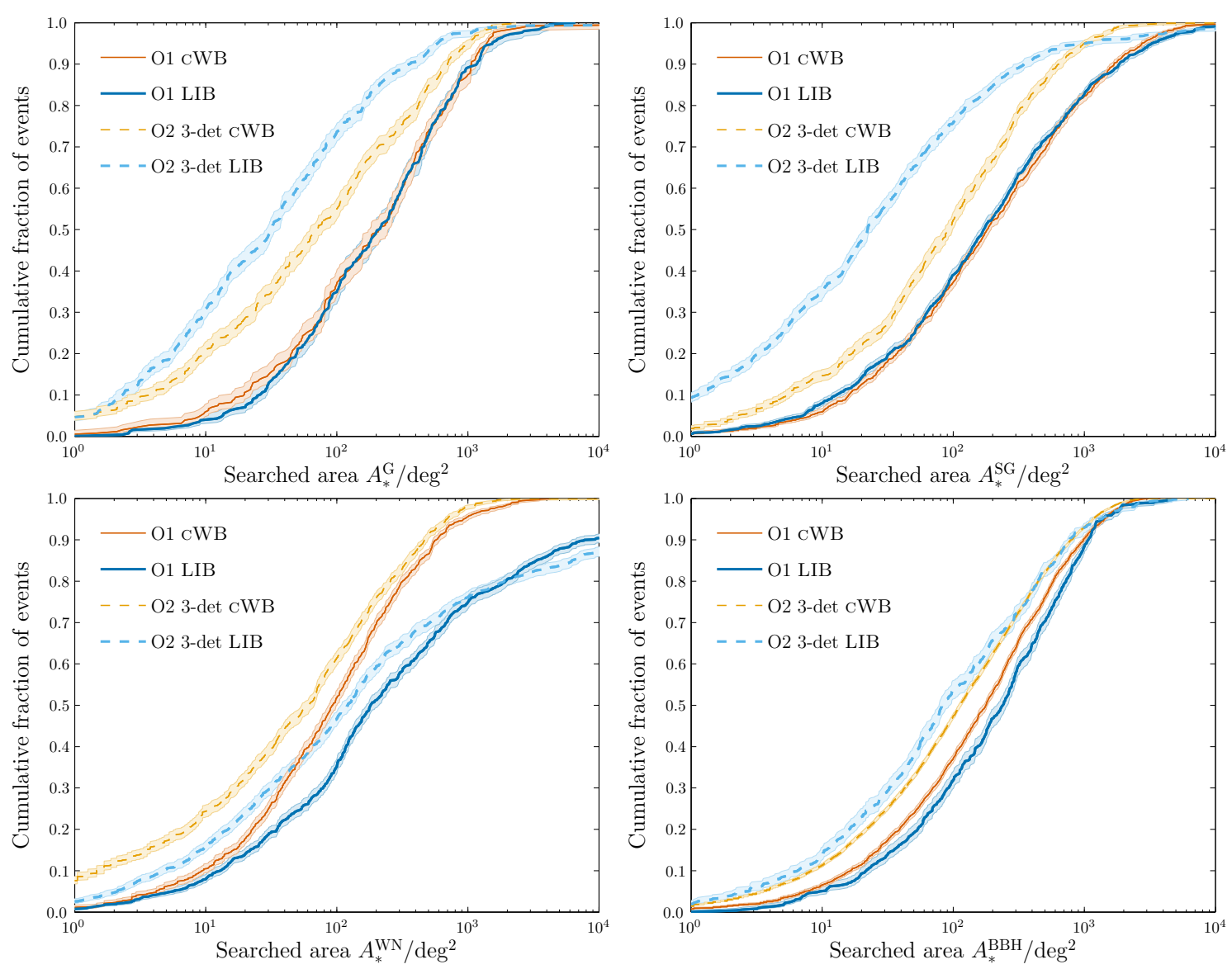

Figure 7: Simulated sky localization for Gaussian (G; top left), sine-Gaussian (SG; top right), broadband white-noise (WN; bottom left) and binary black-hole (BBH; bottom right) bursts during the first two observing runs (O1, see Section 4.1, and O2, see Section 4.2). The plots show the cumulative fractions of events with searched areas $A_{*}$ smaller than the abscissa value. Results are shown for the low-latency COHEREnt WAVEBurst (CWB) [70, 71, 73] and higher-latency LALInfEREnceBurst (LIB) [110] codes. The $\mathrm{O} 2$ results consider only a three-detector (3-det) network; assuming an instrument duty cycle of $\sim 80 \%$, this would be operational $\sim 50 \%$ of the time. The shaded areas indicate the $68 \%$ confidence intervals on the cumulative distributions. A detection threshold of a false alarm rate of approximately $1 \mathrm{yr}^{-1}$ is used and results are taken from [51]. 


\section{Observing Scenario}

In this section we estimate the sensitivity, possible number of detections, and localization capability for each of the observing runs laid out in Section 2.2. We discuss each future observing run in turn and also summarize the results in Table 1.

In the following, we estimate the expected number of BNS coalescence detections using both the lower and upper estimates on the BNS source rate density, $10^{-8}-10^{-5} \mathrm{Mpc}^{-3} \mathrm{yr}^{-1}$ [13]. Given the detectors' noise spectral densities, the $\rho_{c}$ detection threshold can be converted into the (source sky-location and orientation averaged) BNS sensitive detection range $R_{\mathrm{BNS}}[13,20]$. From this, the BNS source rate density can be converted into an estimate of the number of expected detected events; this estimate carries the large error on the source rate density. Similar estimates may be made for NS-BH binaries using the fact that the NS-BH range is approximately a factor of 1.6 larger than the BNS range, ${ }^{5}$ though the uncertainty in the NS-BH source rate density is slightly larger [13]. We assume a nominal $\rho_{c}$ threshold of 12 , at which the expected FAR is $10^{-2} \mathrm{yr}^{-1}$. However, such a stringent threshold may not be appropriate for selecting candidate triggers for electromagnetic follow-up. For example, selecting CBC candidates at thresholds corresponding to a higher background rate of $1 \mathrm{yr}^{-1}\left(100 \mathrm{yr}^{-1}\right)$ would increase the number of true signals subject to electromagnetic follow-up by about 30\% (90\%). The area localization for these low-threshold signals is only fractionally worse than for the high-threshold population - by approximately $20 \%$ $(60 \%)$. The localization of NS-BH signals is expected to be similar to that of BNS signals.

For typical burst sources, the GW waveform is not well known. However, the performance of burst searches is largely independent of the detailed waveform morphology [14, 51], allowing us to quote an approximate sensitive range determined by the total energy $E_{\mathrm{GW}}$ emitted in GWs, the central frequency $f_{0}$ of the burst, the detector noise spectrum $S(f)$, and the single-detector SNR threshold $\rho_{\text {det }}[101]$,

$$
R_{\mathrm{burst}} \simeq\left[\frac{G}{2 \pi^{2} c^{3}} \frac{E_{\mathrm{GW}}}{S\left(f_{0}\right) f_{0}^{2} \rho_{\mathrm{det}}^{2}}\right]^{1 / 2}
$$

In this article, we quote ranges using $E_{\mathrm{GW}}=10^{-2} M_{\odot} c^{2}$ and $f_{0}=150 \mathrm{~Hz} ; E_{\mathrm{GW}}=10^{-2} M_{\odot} c^{2}$ is an optimistic value for GW emission from stellar collapse (see, e.g., [18]), the uncertainty in $E_{\mathrm{GW}}$ means that the quoted burst ranges are more uncertain than their BNS counterparts. We use a single-detector SNR threshold of 8 , corresponding to a typical $\rho_{c} \simeq 12$ and FAR of $\sim 0.3 \mathrm{yr}^{-1}$. Due to the tail of the low-frequency background-rate-vs-amplitude distribution in Figure 3, we see that varying the selection threshold from a background of $0.1 \mathrm{yr}^{-1}\left(\rho_{c} \gtrsim 15\right)$ to even $3 \mathrm{yr}^{-1}$ $\left(\rho_{c} \gtrsim 10\right)$ would increase the number of true signals selected for electromagnetic follow-up by a factor $(15 / 10)^{3} \sim 3$, though the area localization for low-SNR bursts may be particularly challenging.

The run durations discussed below are in calendar time. Based on prior experience, we can reasonably expect a duty cycle of $\sim 80 \%$ for each instrument during observing runs. ${ }^{6}$ Assuming downtime periods are uncorrelated among detectors, this means that all detectors in a three-detector network will be operating in coincidence approximately $50 \%$ of the time and two of the three detectors will be operating an additional $40 \%$ of the time. For a four-detector network, three or more detectors will be operational around $80 \%$ of the time. Our estimates for the expected number of detections and the fraction of sources localized account for these duty cycles. The number of detections also account for the uncertainty in the detector sensitive ranges as indicated in Figure 1.

\footnotetext{
5 This assumes a black-hole mass of $5 M_{\odot}$.

6 This is consistent with what was achieved with the initial detectors, where H1 and L1 had duty cycles of $78 \%$ and $67 \%$ in the S5 observing run [21]. Virgo had duty cycles of $81 \%, 80 \%$ and $73 \%$ in observing runs VSR1-3 respectively [1].
} 


\section{$4.12015-2016$ run (O1): aLIGO 40-80 Mpc}

This is the first advanced-detector observing run, lasting four months, starting 18 September 2015 and ending 12 January 2016. The aLIGO sensitivity is expected to be similar to the early band in Figure 1, with a BNS range of $40-80 \mathrm{Mpc}$, and a burst range of $40-60 \mathrm{Mpc}$ for $E_{\mathrm{GW}}=10^{-2} M_{\odot} c^{2}$. Our experience so far indicates that sensitivity will probably be at the better end of this span, with a BNS range potentially in the interval $60-80 \mathrm{Mpc}$.

A four-month run gives a BNS search volume of $(0.5-4) \times 10^{5} \mathrm{Mpc}^{3} \mathrm{yr}$ at the confident detection threshold of $\rho_{c}=12$. The search volume is $(4 / 3) \pi R^{3} \times T$, where $R$ is the range and $T$ is the observing time incorporating the effects of the detectors' duty cycles. We therefore expect $0.0005-4$ BNS detections. A BNS detection is likely only if the most optimistic astrophysical rates hold.

With the two-detector H1-L1 network any detected events are unlikely to be well localized. A full parameter-estimation study using realistic detector noise and an astrophysically-motivated source catalog has been completed for 2015-2016 [31]. ${ }^{7}$ This used a noise curve in the middle of the early range shown in Figure 1 (the early curve specified in [30]). The distribution of results is shown in Figure 6. It was found that using an SNR threshold of 12, the median $90 \%$ credible region for BNS signals is $\sim 500 \mathrm{deg}^{2}$, and only $4 \%$ of events are expected to have $\mathrm{CR}_{0.9}^{\mathrm{BNS}}$ smaller than $100 \mathrm{deg}^{2}$; the searched area $A_{*}^{\text {BNS }}$ is smaller than $20 \mathrm{deg}^{2}$ for $16 \%$ of events and smaller than $5 \mathrm{deg}^{2}$ in $6 \%$. If a FAR threshold of $10^{-2} \mathrm{yr}^{-1}$ is used without the SNR cut, these localizations change because of the inclusion of an additional population of events with SNRs $\sim 10-12$. The median $\mathrm{CR}_{0.9}^{\mathrm{BNS}}$ is $\sim 600 \mathrm{deg}^{2}$ and $3 \%$ have $\mathrm{CR}_{0.9}^{\mathrm{BNS}}$ smaller than $100 \mathrm{deg}^{2} ; A_{*}^{\mathrm{BNS}}$ is smaller than $20 \mathrm{deg}^{2}$ for $14 \%$ of events and smaller than $5 \mathrm{deg}^{2}$ for $4 \%$.

Equivalent (but not directly comparable) results for bursts are found in [51]. Specific results depend upon the waveform morphology used, but the median searched area is $\sim 1-2$ times larger than for BNS signals; part of this difference is due to the burst study using a less-stringent FAR threshold of $\sim 1 \mathrm{yr}^{-1}$. The distribution of searched areas for four waveform morphologies are shown in Figure 7.

Follow-up observations of a GW signal would be difficult, but not impossible. Localizations provided by another instrument, such as a gamma-ray burst telescope, could improve the possibility of locating an optical or a radio counterpart.

\section{$4.22016-2017$ run (O2): aLIGO 80-120 Mpc, AdV 20-60 Mpc}

This is envisioned to be a six-month run with three detectors. The aLIGO performance is expected to be similar to the mid band in Figure 1, with a BNS range of $80-120 \mathrm{Mpc}$, and a burst range of $60-75 \mathrm{Mpc}$ for $E_{\mathrm{GW}}=10^{-2} M_{\odot} c^{2}$. The AdV range may be similar to the early band, approximately $20-60 \mathrm{Mpc}$ for BNS and 20-40 Mpc for bursts. This gives a BNS search volume of $(0.6-2) \times 10^{6} \mathrm{Mpc}^{3} \mathrm{yr}$, and an expected number of 0.006-20 BNS detections.

Source localization for various points in the sky for CBC signals for the three-detector network is sketched in Figure 8.

BNS sky localization for 2016-2017 (in addition to 2015-2016) has been investigated in [99]. This assumed a noise curve which lies in the middle of the mid range in Figure 1 for aLIGO (the mid curve specified in [30]) and the geometric mean of the upper and lower bounds of the mid region in Figure 1 for AdV. The distribution of results is shown in Figure 6. Performing parameter estimation on an astrophysically-motivated BNS population, with an SNR threshold of 12 (in addition to a FAR cut of $10^{-2} \mathrm{yr}^{-1}$ ), it was found that the median $90 \%$ credible region for BNS signals is $\sim 200 \mathrm{deg}^{2}$, and $20 \%$ of events are expected to have $\mathrm{CR}_{0.9}^{\mathrm{BNS}}$ smaller than $20 \mathrm{deg}^{2}$. The searched area is smaller than $20 \mathrm{deg}^{2}$ for $44 \%$ of events and smaller than $5 \mathrm{deg}^{2}$ for $20 \%$. The burst study [51] gives approximately equivalent results, producing median searched areas a factor

\footnotetext{
7 This study used noise from the sixth science run of initial LIGO, recolored to the expected O1 sensitivity curve. The source catalog, as well as the analysis pipeline, is shared with [99].
} 
of $\sim 2-3$ larger than the BNS results; these results are shown in Figure 7.

\subsection{7-2018 run (O3): aLIGO 120-170 Mpc, AdV 60-85 Mpc}

This is envisioned to be a nine-month run with three detectors. The aLIGO and AdV sensitivities will be similar to the late and mid bands of Figure 1 respectively, with BNS ranges of $120-170 \mathrm{Mpc}$ and $60-85 \mathrm{Mpc}$, and burst ranges of $75-90 \mathrm{Mpc}$ and $40-50 \mathrm{Mpc}$ for $E_{\mathrm{GW}}=10^{-2} M_{\odot} c^{2}$. This gives a BNS search volume of $(3-10) \times 10^{6} \mathrm{Mpc}^{3} \mathrm{yr}$, and an expected $0.04-100$ BNS detections. Source localization for CBC signals is illustrated in Figure 8. While the greater range compared to the 2016-2017 run increases the expected number of detections, the detector bandwidths are marginally smaller. This slightly degrades the localization capability for a source at a fixed SNR.

\section{$4.42019+$ runs: aLIGO $200 \mathrm{Mpc}$, AdV 65-130 Mpc}

At this point we anticipate extended runs with the detectors at or near design sensitivity. The aLIGO detectors are expected to have a sensitivity curve similar to the design curve of Figure 1. AdV may be operating similarly to the late band, eventually reaching the design sensitivity circa 2021. This gives a per-year BNS search volume of $2 \times 10^{7} \mathrm{Mpc}^{3} \mathrm{yr}$, giving an expected $0.2-200$ confident BNS detections annually. Source localization for CBC signals is illustrated in Figure 8. The fraction of signals localized to areas of a few tens of square degrees is greatly increased compared to previous runs. This is due to the much larger detector bandwidths, particularly for AdV; see Figure 1.

\subsection{2 + runs: aLIGO (including India) $200 \mathrm{Mpc}$, AdV $130 \mathrm{Mpc}$}

The four-site network incorporating LIGO-India at design sensitivity would have both improved sensitivity and better localization capabilities. The per-year BNS search volume increases to $4 \times 10^{7} \mathrm{Mpc}^{3} \mathrm{yr}$, giving an expected $0.4-400 \mathrm{BNS}$ detections annually. Source localization is illustrated in Figure 8. The addition of a fourth detector site allows for good source localization over the whole sky [96, 109, 79, 91]. 

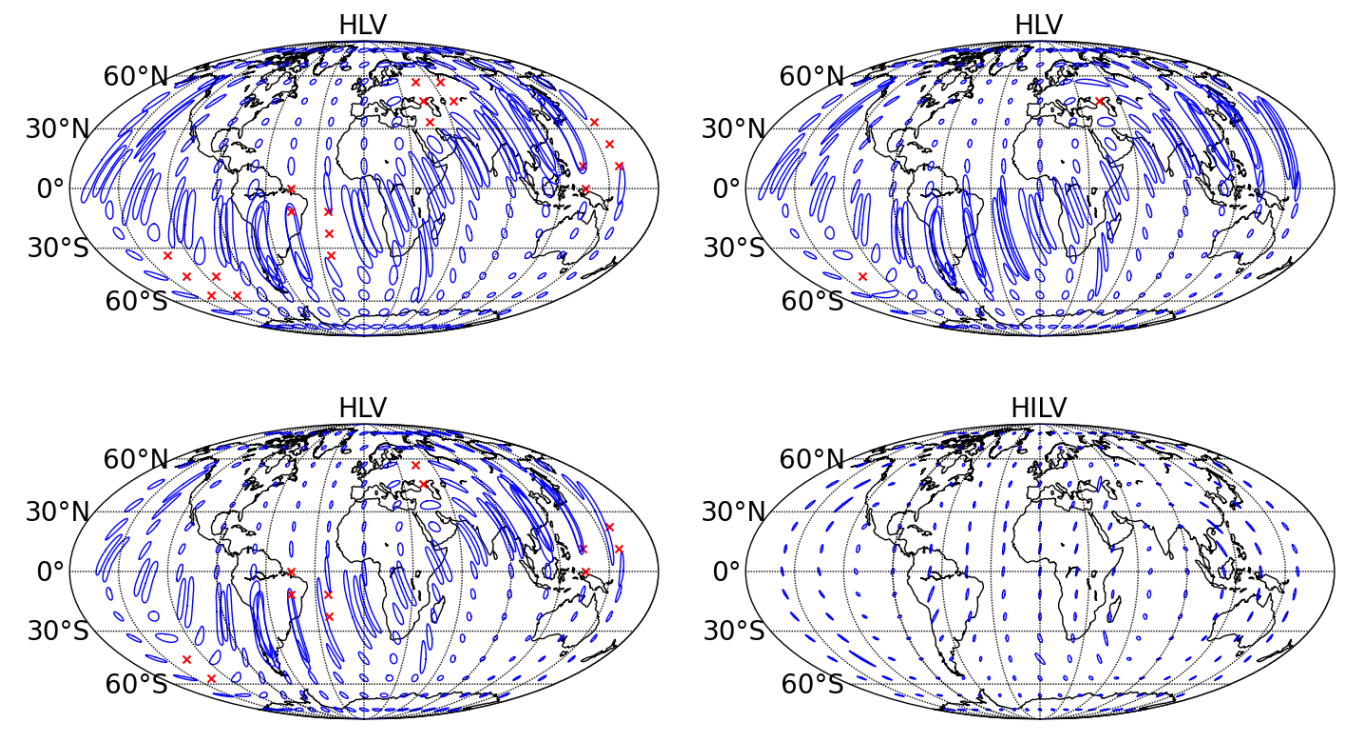

Figure 8: Schematic network sensitivity and localization accuracy for face-on binary neutron-star (BNS) systems with advanced-detector networks. The ellipses show $90 \%$ confidence localization areas based upon timing triangulation alone, and the red crosses show regions of the sky where the signal would not be confidently detected. The top two plots show the localization expected for a BNS system at $80 \mathrm{Mpc}$ by the LIGO Hanford (H)-LIGO Livingston (L)-Virgo (V) network (HLV) in the 2016-2017 run (left) and 2017-2018 run (right). The bottom two plots show the localization expected for a BNS system at $160 \mathrm{Mpc}$ by the HLV network in the 2019+ run (left) and by the four-detector network (HILV) comprising three LIGO sites - in Hanford, Livingston and India (I) - and Virgo operating in 2022+ with all detectors at final design sensitivity $(r i g h t)$. The inclusion of a fourth site in India provides good localization over the whole sky. 
Table 1: Summary of a plausible observing schedule, expected sensitivities, and source localization with the advanced LIGO and Virgo detectors, which will be strongly dependent on the detectors' commissioning progress. The burst ranges assume standard-candle emission of $10^{-2} M_{\odot} c^{2}$ in gravitational waves at $150 \mathrm{~Hz}$ and scale as $E_{\mathrm{GW}}^{1 / 2}$, so it is greater for more energetic sources (such as binary black holes). The binary neutron-star (BNS) localization is characterized by the size of the $90 \%$ credible region (CR) and the searched area. For 2015-2016 and 2016-2017, these have been calculated from parameter-estimation studies (neglecting detector calibration uncertainty) [31, 99] using LALINFEREnCE [110]. The CRs for subsequent periods are estimated from timing triangulation (highlighted by italics), which is known to provide estimates on average a factor of $\sim 4$ too large for a three-detector network [60, 31], hence these serve as a conservative bound. Both ranges as well as the BNS timing-triangulation localizations reflect the uncertainty in the detector noise spectra shown in Figure 1. Differences in the shape of the detector noise curves and also relative sensitivities between detectors have an effect on the localization areas. The BNS detection numbers also account for the uncertainty in the BNS source rate density [13]. BNS detection numbers and localization estimates are computed assuming a signal-to-noise ratio greater than 12 . Burst localizations are expected to be broadly similar to those derived from timing triangulation, but vary depending on the signal bandwidth; the median burst searched area (with a false alarm rate of $\sim 1 \mathrm{yr}^{-1}$ ) may be a factor of $\sim 2-3$ larger than the values quoted for BNS signals [51]. No burst detection numbers are given, since the source rates are currently unknown. Localization and detection numbers assume an $80 \%$ duty cycle for each instrument.

\begin{tabular}{|c|c|c|c|c|c|c|c|}
\hline \multicolumn{3}{|c|}{ Epoch } & $2015-2016$ & $2016-2017$ & $2017-2018$ & $2019+$ & $2022+$ (India) \\
\hline \multicolumn{3}{|c|}{ Estimated run duration } & 4 months & 6 months & 9 months & (per year) & (per year) \\
\hline \multirow{2}{*}{\multicolumn{2}{|c|}{ Burst range/Mpc }} & LIGO & $40-60$ & $60-75$ & $75-90$ & 105 & 105 \\
\hline & & Virgo & - & $20-40$ & $40-50$ & $40-80$ & 80 \\
\hline \multirow{2}{*}{\multicolumn{2}{|c|}{$\mathrm{BNS}$ range/Mpc }} & LIGO & $40-80$ & $80-120$ & $120-170$ & 200 & 200 \\
\hline & & Virgo & - & $20-60$ & $60-85$ & $65-115$ & 130 \\
\hline \multicolumn{3}{|c|}{ Estimated BNS detections } & $0.0005-4$ & $0.006-20$ & $0.04-100$ & $0.2-200$ & $0.4-400$ \\
\hline \multirow{3}{*}{$90 \% \mathrm{CR}$} & \multirow{3}{*}{\multicolumn{2}{|c|}{$\begin{array}{cc}\% \text { within } & 5 \mathrm{deg}^{2} \\
20 \mathrm{deg}^{2} \\
\text { median } / \mathrm{deg}^{2}\end{array}$}} & $<1$ & 2 & $>1-2$ & $>3-8$ & $>20$ \\
\hline & & & $<1$ & 14 & $>10$ & $>8-30$ & $>50$ \\
\hline & & & 480 & 230 & - & - & - \\
\hline \multirow{3}{*}{ searched area } & \multirow{3}{*}{\multicolumn{2}{|c|}{$\begin{array}{cc}\% \text { within } & 5 \operatorname{deg}^{2} \\
20 \operatorname{deg}^{2} \\
\text { median } / \operatorname{deg}^{2}\end{array}$}} & 6 & 20 & - & - & - \\
\hline & & & 16 & 44 & - & - & - \\
\hline & & & 88 & 29 & - & - & - \\
\hline
\end{tabular}




\section{Conclusions}

We have presented a possible observing scenario for the Advanced LIGO and Advanced Virgo network of GW detectors, with emphasis on the expected sensitivities and sky-localization accuracies. This network began operations in September 2015. Unless the most optimistic astrophysical rates hold, two or more detectors with an average range of at least $100 \mathrm{Mpc}$ and with a run of several months will be required for BNS detection.

Electromagnetic follow-up of GW candidates may help confirm GW candidates that would not be confidently identified from GW observations alone. However, such follow-ups would need to deal with large position uncertainties, with areas of many tens to thousands of square degrees. This is likely to remain the situation until late in the decade. Optimizing the electromagnetic follow-up and source identification is an outstanding research topic (see, e.g., [15, 5, 67, 99, 39, 52, 59]). Triggering of focused searches in GW data by electromagnetically-detected events can also help in recovering otherwise hidden GW signals.

Networks with at least two detectors with sensitivities of the order of $200 \mathrm{Mpc}$ are expected to yield detections based purely on GW data after a few years of observation, even under the most pessimistic predictions of signal rates. Sky localization will continue to be poor until a third detector reaches a sensitivity within a factor of $\sim 2$ of the others and with a broad frequency bandwidth. With a four-site detector network at final design sensitivity, we may expect a significant fraction of GW signals to be localized to within a few square degrees by GW observations alone.

The purpose of this article is to provide information to the astronomy community to facilitate planning for multi-messenger astronomy with advanced GW detectors. While the scenarios described here are our best current projections, they will likely evolve as detector installation and commissioning progress. We will therefore update this article regularly. 


\section{Acknowledgements}

The authors gratefully acknowledge the support of the United States National Science Foundation (NSF) for the construction and operation of the LIGO Laboratory and Advanced LIGO as well as the Science and Technology Facilities Council (STFC) of the United Kingdom, the Max-Planck-Society (MPS), and the State of Niedersachsen/Germany for support of the construction of Advanced LIGO and construction and operation of the GEO 600 detector. Additional support for Advanced LIGO was provided by the Australian Research Council. The authors gratefully acknowledge the Italian Istituto Nazionale di Fisica Nucleare (INFN), the French Centre National de la Recherche Scientifique (CNRS) and the Foundation for Fundamental Research on Matter supported by the Netherlands Organisation for Scientific Research, for the construction and operation of the Virgo detector and the creation and support of the EGO consortium. The authors also gratefully acknowledge research support from these agencies as well as by the Council of Scientific and Industrial Research of India, Department of Science and Technology, India, Science \& Engineering Research Board (SERB), India, Ministry of Human Resource Development, India, the Spanish Ministerio de Economía y Competitividad, the Conselleria d'Economia i Competitivitat and Conselleria d'Educació, Cultura i Universitats of the Govern de les Illes Balears, the National Science Centre of Poland, the FOCUS Programme of Foundation for Polish Science, the European Union, the Royal Society, the Scottish Funding Council, the Scottish Universities Physics Alliance, the Lyon Institute of Origins (LIO), the National Research Foundation of Korea, Industry Canada and the Province of Ontario through the Ministry of Economic Development and Innovation, the National Science and Engineering Research Council Canada, the Brazilian Ministry of Science, Technology, and Innovation, the Research Corporation, Ministry of Science and Technology (MOST), Taiwan and the Kavli Foundation. The authors gratefully acknowledge the support of the NSF, STFC, MPS, INFN, CNRS and the State of Niedersachsen/Germany for provision of computational resources.

This article has been assigned LIGO Document number P1200087, Virgo Document number VIR-0288A-12. 


\section{A Changes Between Versions}

Since the arXiv-only first version [4], several updates to the document have been made. The key differences are outlined below.

\section{A.1 Updates to detector commissioning}

The plausible detector scenarios remain largely unchanged, although the text has been updated to reflect our progress. With the aLIGO instruments now completed and both on a path of increasing sensitivity, and AdV advancing, the previous sensitivity evolution appears to remain on track. Modifications that have been made are:

1. While the O1 BNS range 40-80 Mpc has remained unchanged, our experience has led us to suggest a probable range within this interval of $60-80 \mathrm{Mpc}$; the range will not be fully determined until the completion of calibration for the run.

2. The length of $\mathrm{O} 1$ has been extended from three months to four months, taking the run into the early part of 2016. The search volume and expected number of detections have been revised proportionately.

3. The possibility of AdV joining aLIGO for $\mathrm{O} 1$ has been removed; this was always optimistic and none of the results assumed that this would be the case.

4. The potential time-line for LIGO-India has been pushed back. This will remain uncertain until funding is finalized, and forecasting progress on such long timescales is difficult.

5. The AdV 2019+ BNS range in Section 2.2 and Table 1 has been changed to $65-115$ Mpc; this does not reflect a change in the timetable, but is just a clarification that a range of $130 \mathrm{Mpc}$ is not expected until 2021.

A potential time-line for the sensitivity evolution and observing runs of detectors is sketched by Figure 2.

\section{A.2 Updates to sky localization}

In addition to the progress made with regards to the detectors, there have also been advances in data analysis. The text has been updated to include discussions of the parameter-estimation codes that will be used in the upcoming observing runs; Section 3 has been reorganized to try to make this more straight-forward to understand. Specific results that have changed are:

1. The estimated localizations of the O1 (Section 4.1) and O2 (Section 4.2) have been updated to use results of full parameter-estimation studies. Results for these are shown in Figure 6 for BNSs and Figure 7 for bursts. Previously, no numbers were given for O1.

2. Table 1 has been updated to include the results of the $\mathrm{O} 1$ and $\mathrm{O} 2$ studies. Timing triangulation is still used to give an indication of sky localization for later observing runs, as full parameterestimation studies have not yet been completed. These will be updated in the future, but currently serve to give rough (conservative) estimates [60,31]. Parameter-estimation results for later observing runs will be included in the future.

3. Table 1 now also lists both the $90 \%$ credible region and the searched area; previously only the $90 \%$ area was listed.

An example BNS sky map, created using current parameter-estimation techniques, is shown in Figure 5 . 


\section{References}

[1] Aasi, J. et al. (LIGO Scientific Collaboration and Virgo Collaboration), "The characterization of Virgo data and its impact on gravitational-wave searches", Class. Quantum Grav., 29, 155002 (2012). [DOI], [ADS], [arXiv:1203.5613 [gr-qc]]. (Cited on pages 10 and 18.)

[2] Aasi, J. et al. (LIGO Scientific Collaboration and Virgo Collaboration), Open call for partnership for the EM identification and follow-up of $G W$ candidate events, LIGO M1300550-v3 / VIR-0494E-13, (LIGO, Pasadena, CA, 2013). URL (accessed 25 September 2015): https://dcc.ligo.org/LIGO-M1300550-v8/public. (Cited on page 6.)

[3] Aasi, J. et al. (LIGO Scientific Collaboration and Virgo Collaboration), "Parameter estimation for compact binary coalescence signals with the first generation gravitational-wave detector network", Phys. Rev. D, 88, 062001 (2013). [DOI], [ADS], [arXiv:1304.1775 [gr-qc]]. (Cited on page 14.)

[4] Aasi, J. et al. (LIGO Scientific Collaboration and Virgo Collaboration), "Prospects for Localization of Gravitational Wave Transients by the Advanced LIGO and Advanced Virgo Observatories", arXiv, e-print, (2013). [ADS], [arXiv:1304.0670v1 [gr-qc]]. (Cited on pages 5 and 25.)

[5] Aasi, J. et al. (LIGO Scientific Collaboration and Virgo Collaboration), "First Searches for Optical Counterparts to Gravitational-wave Candidate Events", Astrophys. J. Suppl. Ser., 211, 7 (2014). [DOI], [ADS], [arXiv:1310.2314 [astro-ph.IM]]. (Cited on page 23.)

[6] Aasi, J. et al. (LIGO Scientific Collaboration and Virgo Collaboration), "Methods and results of a search for gravitational waves associated with gamma-ray bursts using the GEO600, LIGO, and Virgo detectors", Phys. Rev. D, 89, 122004 (2014). [DOI], [ADS], [arXiv:1405.1053 [astro-ph.HE]]. (Cited on page 13.)

[7] Aasi, J. et al. (LIGO Scientific Collaboration, Virgo Collaboration and IPN Collaboration), "Search for gravitational waves associated with $\gamma$-ray bursts detected by the Interplanetary Network", Phys. Rev. Lett., 113, 011102 (2014). [DOI], [ADS], [arXiv:1403.6639 [astro-ph.HE]]. (Cited on page 13.)

[8] Aasi, J. et al. (LIGO Scientific Collaboration and Virgo Collaboration), The LSC-Virgo White Paper on Gravitational Wave Searches and Astrophysics (2014-2015 edition), LIGO-T1400054-v6, (LIGO, Pasadena, CA, 2014). URL (accessed 23 June 2014): https://dcc.ligo.org/LIGO-T1400054/public. (Cited on pages 5 and 9.)

[9] Aasi, J. et al. (LIGO Scientific Collaboration), "Advanced LIGO", Class. Quantum Grav., 32, 074001 (2015). [DOI], [ADS], [arXiv:1411.4547 [gr-qc]]. (Cited on page 5.)

[10] Aasi, J. et al. (LIGO Scientific Collaboration and Virgo Collaboration), "Characterization of the LIGO detectors during their sixth science run", Class. Quantum Grav., 32, 115012 (2015). [DOI], [ADS], [arXiv:1410.7764 [gr-qc]]. (Cited on page 10.)

[11] Aasi, J. et al. (LIGO Scientific Collaboration), Instrument Science White Paper, LIGO-T1400316-v4, (LIGO, Pasadena, CA, 2015). URL (accessed 28 August 2015): https://dcc.ligo.org/LIGO-T1400316/public. (Cited on page 8.)

[12] Abadie, J. et al. (LIGO Scientific Collaboration and Virgo Collaboration), "All-sky search for gravitational-wave bursts in the first joint LIGO-GEO-Virgo run", Phys. Rev. D, 81, 102001 (2010). [DOI], [ADS], [arXiv:1002.1036 [gr-qc]]. (Cited on page 14.)

[13] Abadie, J. et al. (LIGO Scientific Collaboration and Virgo Collaboration), "Predictions for the rates of compact binary coalescences observable by ground-based gravitational-wave detectors", Class. Quantum Grav., 27, 173001 (2010). [DOI], [ADS], [arXiv:1003.2480 [astro-ph.HE]]. (Cited on pages 6, 9, 18, and 22.) 
[14] Abadie, J. et al. (LIGO Scientific Collaboration and Virgo Collaboration), "All-sky search for gravitational-wave bursts in the second joint LIGO-Virgo run", Phys. Rev. D, 85, 122007 (2012). [DOI], [ADS], [arXiv:1202.2788 [gr-qc]]. (Cited on pages 9, 10, 11, 14, and 18.)

[15] Abadie, J. et al. (LIGO Scientific Collaboration and Virgo Collaboration), "First low-latency LIGO+Virgo search for binary inspirals and their electromagnetic counterparts", Astron. Astrophys., 541, A155 (2012). [DOI], [ADS], [arXiv:1112.6005 [astro-ph.CO]]. (Cited on pages 10 and 23.)

[16] Abadie, J. et al. (LIGO Scientific Collaboration and Virgo Collaboration), "Implementation and testing of the first prompt search for gravitational wave transients with electromagnetic counterparts", Astron. Astrophys., 539, A124 (2012). [DOI], [ADS], [arXiv:1109.3498 [astro-ph.IM]]. (Cited on pages 10, 13, and 16.)

[17] Abadie, J. et al. (LIGO Scientific Collaboration and Virgo Collaboration), LSC and Virgo Policy on Releasing Gravitational Wave Triggers to the Public in the Advanced Detectors Era, LIGO M1200055v2 / VIR-0173A-12, (LIGO, Pasadena, CA, 2012). URL (accessed 16 May 2013): https://dcc.ligo.org/LIGO-M1200055-v2/public. (Cited on page 6.)

[18] Abadie, J. et al. (LIGO Scientific Collaboration and Virgo Collaboration), "Search for Gravitational Waves Associated with Gamma-Ray Bursts during LIGO Science Run 6 and Virgo Science Runs 2 and 3", Astrophys. J., 760, 12 (2012). [DOI], [ADS], [arXiv:1205.2216 [astro-ph.HE]]. (Cited on pages 9,13 , and 18.)

[19] Abadie, J. et al. (LIGO Scientific Collaboration and Virgo Collaboration), "Search for gravitational waves from low mass compact binary coalescence in LIGO's sixth science run and Virgo's science runs 2 and 3", Phys. Rev. D, 85, 082002 (2012). [DOI], [ADS], [arXiv:1111.7314 [gr-qc]]. (Cited on pages 9,10 , and 11.)

[20] Abadie, J. et al. (LIGO Scientific Collaboration and Virgo Collaboration), "Sensitivity Achieved by the LIGO and Virgo Gravitational Wave Detectors during LIGO's Sixth and Virgo's Second and Third Science Runs", arXiv, e-print, (2012). [ADS], [arXiv:1203.2674 [gr-qc]]. (Cited on pages 6 and 18.)

[21] Abbott, B. P. et al. (LIGO Scientific Collaboration), "LIGO: The Laser interferometer gravitationalwave observatory", Rep. Prog. Phys., 72, 076901 (2009). [DOI], [ADS], [arXiv:0711.3041 [gr-qc]]. (Cited on page 18.)

[22] Abbott, B. P. et al. (LIGO Scientific Collaboration), "Search for gravitational-wave bursts in the first year of the fifth LIGO science run", Phys. Rev. D, 80, 102001 (2009). [DOI], [ADS], [arXiv:0905.0020 [gr-qc]]. (Cited on page 11.)

[23] Accadia, T. et al. (Virgo Collaboration), Advanced Virgo Technical Design Report, VIR-0128A-12, (Virgo, Cascina, 2012). URL (accessed 16 May 2013):

https://tds.ego-gw.it/ql/?c=8940. (Cited on pages 5 and 7.)

[24] Acernese, F. et al. (Virgo Collaboration), Advanced Virgo Baseline Design, VIR-027A-09, (Virgo, Cascina, 2009). URL (accessed 16 May 2013):

https://tds.ego-gw.it/ql/?c=6589. (Cited on page 5.)

[25] Acernese, F. et al. (Virgo Collaboration), "Advanced Virgo: a second-generation interferometric gravitational wave detector", Class. Quantum Grav., 32, 024001 (2015). [DOI], [ADS], [arXiv:1408.3978 [gr-qc]]. (Cited on page 5.)

[26] Adams, T. S., Meacher, D., Clark, J., Sutton, P. J., Jones, G. and Minot, A., "Gravitational-wave detection using multivariate analysis", Phys. Rev. D, 88, 062006 (2013). [DOI], [ADS], [arXiv:1305.5714 [gr-qc]]. (Cited on page 9.)

[27] Allen, B., " $\chi^{2}$ time-frequency discriminator for gravitational wave detection", Phys. Rev. D, 71, 062001 (2005). [DOI], [ADS], [arXiv:gr-qc/0405045 [gr-qc]]. (Cited on page 10.) 
[28] Aso, Y., Michimura, Y., Somiya, K., Ando, M., Miyakawa, O., Sekiguchi, T., Tatsumi, D. and Yamamoto, H. (KAGRA Collaboration), "Interferometer design of the KAGRA gravitational wave detector", Phys. Rev. D, 88, 043007 (2013). [DOI], [ADS], [arXiv:1306.6747 [gr-qc]]. (Cited on page 8.)

[29] Babak, S. et al., "Searching for gravitational waves from binary coalescence", Phys. Rev. D, 87, 024033 (2013). [DOI], [ADS], [arXiv:1208.3491 [gr-qc]]. (Cited on page 11.)

[30] Barsotti, L. and Fritschel, P. (LIGO Scientific Collaboration), Early aLIGO Configurations: example scenarios toward design sensitivity, LIGO-T1200307-v4, (LIGO, Pasadena, CA, 2012). URL (accessed 23 June 2014):

https://dcc.ligo.org/LIGO-T1200307/public. (Cited on page 19.)

[31] Berry, C. P. L. et al., "Parameter estimation for binary neutron-star coalescences with realistic noise during the Advanced LIGO era", Astrophys. J., 804, 114 (2015). [DOI], [ADS], [arXiv:1411.6934 [astro-ph.HE]]. (Cited on pages 11, 12, 13, 14, 15, 19, 22, and 25.)

[32] Blackburn, L., Briggs, M. S., Camp, J., Christensen, N., Connaughton, V., Jenke, P., Remillard, R. A. and Veitch, J., "High-energy electromagnetic offline follow-up of LIGO-Virgo gravitationalwave binary coalescence candidate events", Astrophys. J. Suppl. Ser., 217, 8 (2015). [DOI], [ADS], [arXiv:1410.0929 [astro-ph.HE]]. (Cited on page 12.)

[33] Blanchet, L., "Gravitational Radiation from Post-Newtonian Sources and Inspiralling Compact Binaries", Living Rev. Relativity, 17, lrr-2014-2 (2014). [DOI], [ADS], [arXiv:1310.1528 [gr-qc]]. (Cited on page 5.)

[34] Brown, D. A., Harry, I., Lundgren, A. and Nitz, A. H., "Detecting binary neutron star systems with spin in advanced gravitational-wave detectors", Phys. Rev. D, 86, 084017 (2012). [DOI], [ADS], [arXiv:1207.6406 [gr-qc]]. (Cited on pages 9 and 11.)

[35] Buonanno, A., Iyer, B. R., Ochsner, E., Pan, Y. and Sathyaprakash, B. S., "Comparison of postNewtonian templates for compact binary inspiral signals in gravitational-wave detectors", Phys. Rev. $D, \mathbf{8 0}, 084043$ (2009). [DOI], [ADS], [arXiv:0907.0700 [gr-qc]]. (Cited on page 9.)

[36] Canizares, P., Field, S. E., Gair, J., Raymond, V., Smith, R. and Tiglio, M., "Accelerated gravitationalwave parameter estimation with reduced order modeling", Phys. Rev. Lett., 114, 071104 (2015). [DOI], [ADS], [arXiv:1404.6284 [gr-qc]]. (Cited on page 14.)

[37] Canizares, P., Field, S. E., Gair, J. R. and Tiglio, M., "Gravitational wave parameter estimation with compressed likelihood evaluations", Phys. Rev. D, 87, 124005 (2013). [DOI], [ADS], [arXiv:1304.0462 [gr-qc]]. (Cited on page 14.)

[38] Cannon, K., Hanna, C. and Peoples, J., "Likelihood-Ratio Ranking Statistic for Compact Binary Coalescence Candidates with Rate Estimation", arXiv, e-print, (2015). [ADS], [arXiv:1504.04632 [astro-ph.IM]]. (Cited on page 10.)

[39] Cannon, K. et al., "Toward Early-Warning Detection of Gravitational Waves from Compact Binary Coalescence", Astrophys. J., 748, 136 (2012). [DOI], [ADS], [arXiv:1107.2665 [astro-ph.IM]]. (Cited on page 23.)

[40] Chassande-Mottin, E., Miele, M., Mohapatra, S. and Cadonati, L., "Detection of gravitationalwave bursts with chirplet-like template families", Class. Quantum Grav., 27, 194017 (2010). [DOI], [ADS], [arXiv:1005.2876 [gr-qc]]. Proceedings, 14th Workshop on Gravitational wave data analysis (GWDAW-14). (Cited on page 9.)

[41] Chatterji, S., Lazzarini, A., Stein, L., Sutton, P. J., Searle, A. and Tinto, M., "Coherent network analysis technique for discriminating gravitational-wave bursts from instrumental noise", Phys. Rev. D, 74, 082005 (2006). [DOI], [ADS], [arXiv:gr-qc/0605002]. (Cited on page 12.) 
[42] Chen, H.-Y. and Holz, D. E., "Facilitating follow-up of LIGO-Virgo events using rapid sky localization", arXiv, e-print, (2015). [ADS], [arXiv:1509.00055 [astro-ph.IM]]. (Cited on page 14.)

[43] Cornish, N. J. and Littenberg, T. B., "BayesWave: Bayesian Inference for Gravitational Wave Bursts and Instrument Glitches", Class. Quantum Grav., 32, 135012 (2015). [DOI], [ADS], [arXiv:1410.3835 [gr-qc]]. (Cited on pages 9, 11, and 16.)

[44] Cutler, C. and Flanagan, É. É., "Gravitational waves from merging compact binaries: How accurately can one extract the binary's parameters from the inspiral wave form?", Phys. Rev. D, 49, 2658-2697 (1994). [DOI], [ADS], [arXiv:gr-qc/9402014 [gr-qc]]. (Cited on page 14.)

[45] Dal Canton, T., Lundgren, A. P. and Nielsen, A. B., "Impact of precession on aligned-spin searches for neutron-star-black-hole binaries", Phys. Rev. D, 91, 062010 (2015). [DOI], [ADS], [arXiv:1411.6815 [gr-qc]]. (Cited on page 9.)

[46] Dal Canton, T. et al., "Implementing a search for aligned-spin neutron star-black hole systems with advanced ground based gravitational wave detectors", Phys. Rev. D, 90, 082004 (2014). [DOI], [ADS], [arXiv:1405.6731 [gr-qc]]. (Cited on page 11.)

[47] de Mink, S. E. and Belczynski, K., "Merger rates of double neutron stars and stellar origin black holes: The Impact of Initial Conditions on Binary Evolution Predictions", Astrophys. J., 814, 58 (2015). [DOI], [ADS], [arXiv:1506.03573 [astro-ph.HE]]. (Cited on page 9.)

[48] Dimmelmeier, H., Ott, C. D., Marek, A. and Janka, H.-T., "Gravitational wave burst signal from the core collapse of rotating stars", Phys. Rev. D, 78, 064056 (2008). [DOI], [ADS], [arXiv:0806.4953 [astro-ph]]. (Cited on page 13.)

[49] Dominik, M., Belczynski, K., Fryer, C., Holz, D. E., Berti, E., Bulik, T., Mandel, I. and O'Shaughnessy, R., "Double Compact Objects II: Cosmological Merger Rates", Astrophys. J., 779, 72 (2013). [DOI], [ADS], [arXiv:1308.1546 [astro-ph.HE]]. (Cited on page 9.)

[50] Dominik, M. et al., "Double Compact Objects III: Gravitational Wave Detection Rates", Astrophys. J., 806, 263 (2015). [DOI], [ADS], [arXiv:1405.7016 [astro-ph.HE]]. (Cited on page 9.)

[51] Essick, R., Vitale, S., Katsavounidis, E., Vedovato, G. and Klimenko, S., "Localization of short duration gravitational-wave transients with the early advanced LIGO and Virgo detectors", Astrophys. J., 800, 81 (2015). [DOI], [ADS], [arXiv:1409.2435 [astro-ph.HE]]. (Cited on pages 13, 14, 16, 17, 18, 19, and 22.)

[52] Evans, P. A. et al., "Optimisation of the Swift X-ray follow-up of Advanced LIGO and Virgo gravitational wave triggers in 2015-16", Mon. Not. R. Astron. Soc., 455, 1522-1537 (2016). [DOI], [ADS], [arXiv:1506.01624 [astro-ph.HE]]. (Cited on page 23.)

[53] Evans, P. A. et al. (LIGO Scientific Collaboration and Virgo Collaboration), "Swift Follow-up Observations of Candidate Gravitational-Wave Transient Events", Astrophys. J. Suppl. Ser., 203, 28 (2012). [DOI], [ADS], [arXiv:1205.1124 [astro-ph.HE]]. (Cited on page 10.)

[54] Faber, J. A. and Rasio, F. A., "Binary Neutron Star Mergers", Living Rev. Relativity, 15, lrr-2012-8 (2012). [DOI], [ADS], [arXiv:1204.3858 [gr-qc]]. (Cited on page 9.)

[55] Fairhurst, S., "Triangulation of gravitational wave sources with a network of detectors", New J. Phys., 11, 123006 (2009). [DOI], [ADS], [arXiv:0908.2356 [gr-qc]]. (Cited on page 11.)

[56] Fairhurst, S., "Source localization with an advanced gravitational wave detector network", Class. Quantum Grav., 28, 105021 (2011). [DOI], [ADS], [arXiv:1010.6192 [gr-qc]]. (Cited on page 11.)

[57] Farr, B. et al., "Parameter estimation on gravitational waves from neutron-star binaries with spinning components", arXiv, e-print, (2015). [ADS], [arXiv:1508.05336 [astro-ph.HE]]. (Cited on page 14.) 
[58] Finn, L. S. and Chernoff, D. F., "Observing binary inspiral in gravitational radiation: One interferometer", Phys. Rev. D, 47, 2198-2219 (1993). [DOI], [ADS], [arXiv:gr-qc/9301003]. (Cited on page 6.)

[59] Gehrels, N., Cannizzo, J. K., Kanner, J., Kasliwal, M. M., Nissanke, S. and Singer, L. P., "Galaxy Strategy for LIGO-Virgo Gravitational Wave Counterpart Searches", arXiv, e-print, (2015). [ADS], [arXiv:1508.03608 [astro-ph.HE]]. (Cited on page 23.)

[60] Grover, K., Fairhurst, S., Farr, B. F., Mandel, I., Rodriguez, C., Sidery, T. and Vecchio, A., "Comparison of Gravitational Wave Detector Network Sky Localization Approximations", Phys. Rev. $D, \mathbf{8 9}, 042004$ (2014). [DOI], [ADS], [arXiv:1310.7454 [gr-qc]]. (Cited on pages 12, 22, and 25.)

[61] Harry, G. M. (LIGO Scientific Collaboration), "Advanced LIGO: the next generation of gravitational wave detectors", Class. Quantum Grav., 27, 084006 (2010). [DOI], [ADS]. (Cited on page 5.)

[62] Harry, I. W., Nitz, A. H., Brown, D. A., Lundgren, A. P., Ochsner, E. and Keppel, D., "Investigating the effect of precession on searches for neutron-star-black-hole binaries with Advanced LIGO", Phys. Rev. D, 89, 024010 (2014). [DOI], [ADS], [arXiv:1307.3562 [gr-qc]]. (Cited on page 9.)

[63] Hild, S. et al. (LIGO Scientific Collaboration), LIGO 3 Strawman Design, T. Red, LIGO-T1200046-v1, (LIGO, Pasadena, C.A, 2012). URL (accessed 1 August 2014): https://dcc.ligo.org/LIGO-T1200046/public. (Cited on page 8.)

[64] Iyer, B. R., Souradeep, T., Unnikrishnan, C. S., Dhurandhar, S., Raja, S. and Sengupta, A. (IndIGO Consortium), LIGO-India, M1100296-v2, (IndIGO, India, 2011). URL (accessed 27 August 2015): https://dcc.ligo.org/LIGO-M1100296/public. (Cited on page 7.)

[65] Jaranowski, P. and Królak, A., "Gravitational-Wave Data Analysis. Formalism and Sample Applications: The Gaussian Case", Living Rev. Relativity, 15, lrr-2012-4 (2012). [DOI], [ADS], [arXiv:0711.1115 [gr-qc]]. (Cited on page 14.)

[66] Kanner, J. B. et al., "Leveraging waveform complexity for confident detection of gravitational waves", Phys. Rev. D, 93, 022002 (2016). [DOI], [ADS], [arXiv:1509.06423 [astro-ph.IM]]. (Cited on pages 9 and 11.)

[67] Kasliwal, Mansi M. and Nissanke, Samaya, "On Discovering Electromagnetic Emission from Neutron Star Mergers: The Early Years of Two Gravitational Wave Detectors", Astrophys. J., 789, L5 (2014). [DOI], [ADS], [arXiv:1309.1554 [astro-ph.HE]]. (Cited on page 23.)

[68] Khan, S., Husa, S., Hannam, M., Ohme, F., Pürrer, M., Forteza, X. J. and Bohé, A., "Frequencydomain gravitational waves from non-precessing black-hole binaries. II. A phenomenological model for the advanced detector era", Phys. Rev. D, 93, 044007 (2016). [DOI], [ADS], [arXiv:1508.07253 [gr-qc]]. (Cited on page 9.)

[69] Kim, C., Perera, B. B. P. and McLaughlin, M. A., "Implications of PSR J0737-3039B for the Galactic NS-NS Binary Merger Rate", Mon. Not. R. Astron. Soc., 448, 928-938 (2015). [DOI], [ADS], [arXiv:1308.4676 [astro-ph.SR]]. (Cited on page 9.)

[70] Klimenko, S., Mohanty, S., Rakhmanov, M. and Mitselmakher, G., "Constraint likelihood analysis for a network of gravitational wave detectors", Phys. Rev. D, 72, 122002 (2005). [DOI], [ADS], [arXiv:gr-qc/0508068 [gr-qc]]. (Cited on pages 14 and 17.)

[71] Klimenko, S.., Yakushin, I.., Mercer, A.. and Mitselmakher, G., "Coherent method for detection of gravitational wave bursts", Class. Quantum Grav., 25, 114029 (2008). [DOI], [ADS], [arXiv:0802.3232 [gr-qc]]. (Cited on pages 9, 14, and 17.)

[72] Klimenko, S. et al., "Localization of gravitational wave sources with networks of advanced detectors", Phys. Rev. D, 83, 102001 (2011). [DOI], [ADS], [arXiv:1101.5408 [astro-ph.IM]]. (Cited on pages 8, 13, and 14.) 
[73] Klimenko, S. et al., "Method for detection and reconstruction of gravitational wave transients with networks of advanced detectors", arXiv, e-print, (2015). [ADS], [arXiv:1511.05999 [gr-qc]]. (Cited on pages 14 and 17.)

[74] Lindblom, L., Owen, B. J. and Brown, D. A., "Model waveform accuracy standards for gravitational wave data analysis", Phys. Rev. D, 78, 124020 (2008). [DOI], [ADS], [arXiv:0809.3844 [gr-qc]]. (Cited on page 9.)

[75] Littenberg, T. B. and Cornish, N. J., "Bayesian inference for spectral estimation of gravitational wave detector noise", Phys. Rev. D, 91, 084034 (2015). [DOI], [ADS], [arXiv:1410.3852 [gr-qc]]. (Cited on page 16.)

[76] Lück, H. et al., "The upgrade of GEO 600", J. Phys.: Conf. Ser., 228, 012012 (2010). [DOI], [ADS], [arXiv:1004.0339 [gr-qc]]. (Cited on page 8.)

[77] Mandel, I. and O'Shaughnessy, R., "Compact Binary Coalescences in the Band of Groundbased Gravitational-Wave Detectors", Class. Quantum Grav., 27, 114007 (2010). [DOI], [ADS], [arXiv:0912.1074 [astro-ph.HE]]. Proceedings, 3rd Annual Meeting, NRDA 2009, Potsdam, Germany, July $6-9,2009$. (Cited on page 14.)

[78] Miller, J., Barsotti, L., Vitale, S., Fritschel, P., Evans, M. and Sigg, D., "Prospects for doubling the range of Advanced LIGO", Phys. Rev. D, 91, 062005 (2015). [DOI], [ADS], [arXiv:1410.5882 [gr-qc]]. (Cited on page 8.)

[79] Nissanke, S., Kasliwal, M. and Georgieva, A., "Identifying Elusive Electromagnetic Counterparts to Gravitational Wave Mergers: An End-to-end Simulation", Astrophys. J., 767, 124 (2013). [DOI], [ADS], [arXiv:1210.6362 [astro-ph.HE]]. (Cited on pages 8, 11, 12, and 20.)

[80] Nissanke, S., Sievers, J., Dalal, N. and Holz, D. E., "Localizing Compact Binary Inspirals on the Sky Using Ground-based Gravitational Wave Interferometers", Astrophys. J., 739, 99 (2011). [DOI], [ADS], [arXiv:1105.3184 [astro-ph.CO]]. (Cited on page 11.)

[81] Nitz, A. H., Lundgren, A., Brown, D. A., Ochsner, E., Keppel, D. and Harry, I. W., "Accuracy of gravitational waveform models for observing neutron-star-black-hole binaries in Advanced LIGO", Phys. Rev. D, 88, 124039 (2013). [DOI], [ADS], [arXiv:1307.1757 [gr-qc]]. (Cited on page 9.)

[82] Ott, C. D., "The gravitational-wave signature of core-collapse supernovae", Class. Quantum Grav., 26, 063001 (2009). [DOI], [ADS], [arXiv:0809.0695 [astro-ph]]. (Cited on page 13.)

[83] Ott, C. D. et al., "Dynamics and gravitational wave signature of collapsar formation", Phys. Rev. Lett., 106, 161103 (2011). [DOI], [ADS], [arXiv:1012.1853 [astro-ph.HE]]. (Cited on page 13.)

[84] Owen, B. J. and Sathyaprakash, B. S., "Matched filtering of gravitational waves from inspiraling compact binaries: Computational cost and template placement", Phys. Rev. D, 60, 022002 (1999). [DOI], [ADS], [arXiv:gr-qc/9808076 [gr-qc]]. (Cited on page 11.)

[85] Pan, Y., Buonanno, A., Taracchini, A., Kidder, L. E., Mroué, A. H., Pfeiffer, H. P., Scheel, M. A. and Szilágyi, B., "Inspiral-merger-ringdown waveforms of spinning, precessing black-hole binaries in the effective-one-body formalism", Phys. Rev. D, 89, 084006 (2014). [DOI], [ADS], [arXiv:1307.6232 [gr-qc]]. (Cited on page 9.)

[86] Pankow, C., Klimenko, S., Mitselmakher, G., Yakushin, I., Vedovato, G., Drago, M., Mercer, R. A. and Ajith, P., "A Burst search for gravitational waves from binary black holes", Class. Quantum Grav., 26, 204004 (2009). [DOI], [ADS], [arXiv:0905.3120 [gr-qc]]. Proceedings, 13th Gravitational Wave Data Analysis Workshop on Bridging gravitational wave astronomy and observational astrophysics (GWDAW13). (Cited on page 11.)

[87] Pitkin, M., Reid, S., Rowan, S. and Hough, J., "Gravitational Wave Detection by Interferometry (Ground and Space)", Living Rev. Relativity, 14, lrr-2011-5 (2011). [DOI], [ADS], [arXiv:1102.3355 [astro-ph.IM]]. (Cited on page 5.) 
[88] Privitera, S. et al., "Improving the sensitivity of a search for coalescing binary black holes with nonprecessing spins in gravitational wave data", Phys. Rev. D, 89, 024003 (2014). [DOI], [ADS], [arXiv:1310.5633 [gr-qc]]. (Cited on page 11.)

[89] Pürrer, M., "Frequency domain reduced order models for gravitational waves from aligned-spin compact binaries", Class. Quantum Grav., 31, 195010 (2014). [DOI], [ADS], [arXiv:1402.4146 [gr-qc]]. (Cited on page 14.)

[90] Read, J. S. et al., "Matter effects on binary neutron star waveforms", Phys. Rev. D, 88, 044042 (2013). [DOI], [ADS], [arXiv:1306.4065 [gr-qc]]. (Cited on page 9.)

[91] Rodriguez, C. L., Farr, B., Raymond, V., Farr, W. M., Littenberg, T. B., Fazi, D. and Kalogera, V., "Basic Parameter Estimation of Binary Neutron Star Systems by the Advanced LIGO/Virgo Network", Astrophys. J., 784, 119 (2014). [DOI], [ADS], [arXiv:1309.3273 [astro-ph.HE]]. (Cited on pages 8,14 , and 20.)

[92] Rodriguez, C. L., Morscher, M., Pattabiraman, B., Chatterjee, S., Haster, C.-J. and Rasio, F. A., "Binary Black Hole Mergers from Globular Clusters: Implications for Advanced LIGO", Phys. Rev. Lett., 115, 051101 (2015). [DOI], [ADS], [arXiv:1505.00792 [astro-ph.HE]]. (Cited on page 9.)

[93] Sathyaprakash, B. et al., "Scientific Objectives of Einstein Telescope", Class. Quantum Grav., 29, 124013 (2012). [DOI], [ADS], [arXiv:1206.0331 [gr-qc]]. (Cited on page 8.)

[94] Sathyaprakash, B. S. and Schutz, B. F., "Physics, Astrophysics and Cosmology with Gravitational Waves", Living Rev. Relativity, 12, lrr-2009-2 (2009). [DOI], [ADS], [arXiv:0903.0338 [gr-qc]]. (Cited on page 5.)

[95] Schmidt, P., Ohme, F. and Hannam, M., "Towards models of gravitational waveforms from generic binaries II: Modelling precession effects with a single effective precession parameter", Phys. Rev. D, 91, 024043 (2015). [DOI], [ADS], [arXiv:1408.1810 [gr-qc]]. (Cited on page 9.)

[96] Schutz, B. F., "Networks of gravitational wave detectors and three figures of merit", Class. Quantum Grav., 28, 125023 (2011). [DOI], [ADS], [arXiv:1102.5421 [astro-ph.IM]]. (Cited on pages 8, 14, and 20.)

[97] Sidery, T. et al., "Reconstructing the sky location of gravitational-wave detected compact binary systems: methodology for testing and comparison", Phys. Rev. D, 89, 084060 (2014). [DOI], [ADS], [arXiv:1312.6013 [astro-ph.IM]]. (Cited on page 14.)

[98] Singer, L. P. and Price, L. R., "Rapid Bayesian position reconstruction for gravitational-wave transients", Phys. Rev. D, 93, 024013 (2016). [DOI], [ADS], [arXiv:1508.03634 [gr-qc]]. (Cited on pages 11, 13, 14, and 15.)

[99] Singer, L. P. et al., "The First Two Years of Electromagnetic Follow-Up with Advanced LIGO and Virgo", Astrophys. J., 795, 105 (2014). [DOI], [ADS], [arXiv:1404.5623 [astro-ph.HE]]. (Cited on pages $11,12,13,14,15,19,22$, and 23.)

[100] Somiya, K. (KAGRA Collaboration), "Detector configuration of KAGRA - the Japanese cryogenic gravitational-wave detector", Class. Quantum Grav., 29, 124007 (2012). [DOI], [ADS], [arXiv:1111.7185 [gr-qc]]. (Cited on page 8.)

[101] Sutton, P. J., "A Rule of Thumb for the Detectability of Gravitational-Wave Bursts", arXiv, e-print, (2013). [ADS], [arXiv:1304.0210 [gr-qc]]. (Cited on page 18.)

[102] Sutton, P. J. et al., "X-Pipeline: An Analysis package for autonomous gravitational-wave burst searches", New J. Phys., 12, 053034 (2010). [DOI], [ADS], [arXiv:0908.3665 [gr-qc]]. (Cited on page 9.) 
[103] Taracchini, A. et al., "Effective-one-body model for black-hole binaries with generic mass ratios and spins", Phys. Rev. D, 89, 061502 (2014). [DOI], [ADS], [arXiv:1311.2544 [gr-qc]]. (Cited on page 9.)

[104] Thrane, E. and Coughlin, M., "Searching for gravitational-wave transients with a qualitative signal model: seedless clustering strategies", Phys. Rev. D, 88, 083010 (2013). [DOI], [ADS], [arXiv:1308.5292 [astro-ph.IM]]. (Cited on page 9.)

[105] Thrane, E., Mandic, V. and Christensen, N., "Detecting very long-lived gravitational-wave transients lasting hours to weeks", Phys. Rev. D, 91, 104021 (2015). [DOI], [ADS], [arXiv:1501.06648 [astroph.IM]]. (Cited on page 9.)

[106] Thrane, E. et al., "Long gravitational-wave transients and associated detection strategies for a network of terrestrial interferometers", Phys. Rev. D, 83, 083004 (2011). [DOI], [ADS], [arXiv:1012.2150 [astro-ph.IM]]. (Cited on page 9.)

[107] Usman, S. A. et al., "An improved pipeline to search for gravitational waves from compact binary coalescence", arXiv, e-print, (2015). [ADS], [arXiv:1508.02357 [gr-qc]]. (Cited on page 10.)

[108] Vallisneri, M., Kanner, J., Williams, R., Weinstein, A. and Stephens, B., "The LIGO Open Science Center", J. Phys.: Conf. Ser., 610, 012021 (2015). [DOI], [ADS], [arXiv:1410.4839 [gr-qc]]. Proceedings, 10th International LISA Symposium. (Cited on page 11.)

[109] Veitch, J. et al., "Estimating parameters of coalescing compact binaries with proposed advanced detector networks", Phys. Rev. D, 85, 104045 (2012). [DOI], [ADS], [arXiv:1201.1195 [astro-ph.HE]]. (Cited on pages $8,11,12,14$, and 20.)

[110] Veitch, J. et al., "Parameter estimation for compact binaries with ground-based gravitational-wave observations using the LALInference software library", Phys. Rev. D, 91, 042003 (2015). [DOI], [ADS], [arXiv:1409.7215 [gr-qc]]. (Cited on pages 11, 13, 14, 15, 16, 17, and 22.)

[111] Vitale, S., Del Pozzo, W., Li, T. G. F., Van Den Broeck, C., Mandel, I., Aylott, B. and Veitch, J., "Effect of calibration errors on Bayesian parameter estimation for gravitational wave signals from inspiral binary systems in the advanced detectors era", Phys. Rev. D, 85, 064034 (2012). [DOI], [ADS], [arXiv:1111.3044 [gr-qc]]. (Cited on page 11.)

[112] Vitale, S. and Zanolin, M., "Application of asymptotic expansions for maximum likelihood estimators' errors to gravitational waves from inspiraling binary systems: The network case", Phys. Rev. D, 84, 104020 (2011). [DOI], [ADS], [arXiv:1108.2410 [gr-qc]]. (Cited on page 11.)

[113] Yakunin, K. N. et al., "Gravitational waves from core collapse supernovae", Class. Quantum Grav., 27, 194005 (2002). [DOI], [ADS], [arXiv:1005.0779 [gr-qc]]. (Cited on page 13.) 


\section{LIGO Scientific Collaboration and Virgo Collaboration}

B. P. Abbott, ${ }^{1}$ R. Abbott, ${ }^{1}$ T. D. Abbott, ${ }^{2}$ M. R. Abernathy, ${ }^{1}$ F. Acernese,${ }^{3,4}$ K. Ackley, ${ }^{5}$ C. Adams,${ }^{6}$ T. Adams,${ }^{7}$ P. Addesso, ${ }^{8}$ R. X. Adhikari, ${ }^{1}$ V. B. Adya,${ }^{9}$ C. Affeldt,${ }^{9}$ M. Agathos,${ }^{10}$ K. Agatsuma, ${ }^{10}$ N. Aggarwal, ${ }^{11}$ O. D. Aguiar, ${ }^{12}$ A. Ain, ${ }^{13}$ P. Ajith, ${ }^{14}$ B. Allen, ${ }^{9,15,16}$ A. Allocca, ${ }^{17,18}$ P. A. Altin ${ }^{19}$ D. V. Amariutei ${ }^{5}$ S. B. Anderson, ${ }^{1}$ W. G. Anderson, ${ }^{15}$ K. Arai, ${ }^{1}$ M. C. Araya, ${ }^{1}$ C. C. Arceneaux, ${ }^{20}$ J. S. Areeda,${ }^{21}$ N. Arnaud ${ }^{22}$ K. G. Arun ${ }^{23}$ G. Ashton, ${ }^{24}$ M. Ast,${ }^{25}$ S. M. Aston, ${ }^{6}$ P. Astone,${ }^{26}$ P. Aufmuth, ${ }^{16}$ C. Aulbert,${ }^{9}$ S. Babak,${ }^{27}$ P. T. Baker,${ }^{28}$ F. Baldaccini, ${ }^{29,30}$ G. Ballardin, ${ }^{31}$ S. W. Ballmer, ${ }^{32}$ J. C. Barayoga,${ }^{1}$ S. E. Barclay,${ }^{33}$ B. C. Barish, ${ }^{1}$ D. Barker,${ }^{34}$ F. Barone, ${ }^{3,4}$ B. Barr, ${ }^{33}$ L. Barsotti, ${ }^{11}$ M. Barsuglia, ${ }^{35}$ D. Barta,${ }^{36}$ J. Bartlett,${ }^{34}$ I. Bartos, ${ }^{37}$ R. Bassiri, ${ }^{38}$ A. Basti, ${ }^{17,18}$ J. C. Batch, ${ }^{34}$ C. Baune, ${ }^{9}$ V. Bavigadda, ${ }^{31}$ M. Bazzan, ${ }^{39,40}$ B. Behnke, ${ }^{27}$ M. Bejger,${ }^{41}$ C. Belczynski, ${ }^{42}$ A. S. Bell, ${ }^{33}$ C. J. Bell, ${ }^{33}$ B. K. Berger, ${ }^{1}$ J. Bergman, ${ }^{34}$ G. Bergmann, ${ }^{9}$ C. P. L. Berry, ${ }^{43}$ D. Bersanetti, ${ }^{44,45}$ A. Bertolini, ${ }^{10}$ J. Betzwieser, ${ }^{6}$ S. Bhagwat, ${ }^{32}$ R. Bhandare, ${ }^{46}$ I. A. Bilenko, ${ }^{47}$ G. Billingsley, ${ }^{1}$ J. Birch ${ }^{6}$ R. Birney, ${ }^{48}$ S. Biscans, ${ }^{11}$ A. Bisht,${ }^{9,16}$ M. Bitossi, ${ }^{31}$ C. Biwer,${ }^{32}$ M. A. Bizouard ${ }^{22}$ J. K. Blackburn, ${ }^{1}$ C. D. Blair,${ }^{49}$ D. Blair ${ }^{49}$ R. M. Blair ${ }^{34}$ S. Bloemen, ${ }^{10,50}$ O. Bock,${ }^{9}$ T. P. Bodiya, ${ }^{11}$ M. Boer,${ }^{51}$ G. Bogaert,${ }^{51}$ C. Bogan,${ }^{9}$ A. Bohe,${ }^{27}$ P. Bojtos,${ }^{52}$ C. Bond,${ }^{43}$ F. Bondu, ${ }^{53}$ R. Bonnand,${ }^{7}$ R. Bork,${ }^{1}$ V. Boschi, ${ }^{18,17}$ S. Bose,${ }^{54,13}$ A. Bozzi, ${ }^{31}$ C. Bradaschia, ${ }^{18}$ P. R. Brady, ${ }^{15}$ V. B. Braginsky, ${ }^{47}$ M. Branchesi, ${ }^{55,56}$ J. E. Brau, ${ }^{57}$ T. Briant, ${ }^{58}$ A. Brillet,${ }^{51}$ M. Brinkmann, ${ }^{9}$ V. Brisson,${ }^{22}$ P. Brockill, ${ }^{15}$ A. F. Brooks,${ }^{1}$ D. A. Brown,${ }^{32}$ D. D. Brown,${ }^{43}$ N. M. Brown, ${ }^{11}$ C. C. Buchanan, ${ }^{2}$ A. Buikema, ${ }^{11}$ T. Bulik, ${ }^{42}$ H. J. Bulten,${ }^{59,10}$ A. Buonanno, ${ }^{27,60}$ D. Buskulic, ${ }^{7}$ C. Buy, ${ }^{35}$ R. L. Byer, ${ }^{38}$ L. Cadonati, ${ }^{61}$ G. Cagnoli, ${ }^{62}$ C. Cahillane, ${ }^{1}$ J. Calderón Bustillo, ${ }^{63,61}$ T. Callister, ${ }^{1}$ E. Calloni, ${ }^{64,4}$ J. B. Camp, ${ }^{65}$ K. C. Cannon, ${ }^{66}$ J. Cao, ${ }^{67}$ C. D. Capano, ${ }^{9}$ E. Capocasa, ${ }^{35}$ F. Carbognani, ${ }^{31}$ S. Caride, ${ }^{68}$ J. Casanueva Diaz ${ }^{22}$ C. Casentini, ${ }^{69,70}$ S. Caudill, ${ }^{15}$ M. Cavaglià ${ }^{20}$ F. Cavalier, ${ }^{22}$ R. Cavalieri, ${ }^{31}$ G. Cella, ${ }^{18}$ C. Cepeda, ${ }^{1}$ L. Cerboni Baiardi, ${ }^{55,56}$ G. Cerretani, ${ }^{17,18}$ E. Cesarini, ${ }^{69,70}$ R. Chakraborty, ${ }^{1}$ T. Chalermsongsak, ${ }^{1}$ S. J. Chamberlin, ${ }^{15}$ M. Chan, ${ }^{33}$ S. Chao, ${ }^{71}$ P. Charlton, ${ }^{72}$ E. Chassande-Mottin, ${ }^{35}$ H. Y. Chen, ${ }^{73}$ Y. Chen, ${ }^{74}$ C. Cheng, ${ }^{71}$ A. Chincarini, ${ }^{45}$ A. Chiummo, ${ }^{31}$ H. S. Cho, ${ }^{75}$ M. Cho, ${ }^{60}$ J. H. Chow, ${ }^{19}$ N. Christensen, ${ }^{76}$ Q. Chu, ${ }^{49}$ S. Chua, ${ }^{58}$ S. Chung, ${ }^{49}$ G. Ciani, ${ }^{5}$ F. Clara, ${ }^{34}$ J. A. Clark, ${ }^{61}$ F. Cleva, ${ }^{51}$ E. Coccia, ${ }^{69,77}$ P.F. Cohadon, ${ }^{58}$ A. Colla, ${ }^{78,26}$ C. G. Collette, ${ }^{79}$ M. Constancio Jr., ${ }^{12}$ A. Conte, ${ }^{78,26}$ L. Conti, ${ }^{40}$ D. Cook, ${ }^{34}$ T. R. Corbitt, ${ }^{2}$ N. Cornish, ${ }^{28}$ A. Corsi,${ }^{80}$ S. Cortese,${ }^{31}$ C. A. Costa, ${ }^{12}$ M. W. Coughlin, ${ }^{76}$ S. B. Coughlin, ${ }^{81}$ J.-P. Coulon, ${ }^{51}$ S. T. Countryman, ${ }^{37}$ P. Couvares, ${ }^{1}$ D. M. Coward, ${ }^{49}$ M. J. Cowart, ${ }^{6}$ D. C. Coyne,${ }^{1}$ R. Coyne, ${ }^{80}$ K. Craig, ${ }^{33}$ J. D. E. Creighton, ${ }^{15}$ J. Cripe, ${ }^{2}$ S. G. Crowder, ${ }^{82}$ A. Cumming, ${ }^{33}$ L. Cunningham, ${ }^{33}$ E. Cuoco, ${ }^{31}$ T. Dal Canton, ${ }^{9}$ S. L. Danilishin, ${ }^{33}$ S. D'Antonio, ${ }^{70}$ K. Danzmann, ${ }^{16,9}$ N. S. Darman, ${ }^{83}$ V. Dattilo, ${ }^{31}$ I. Dave, ${ }^{46}$ H. P. Daveloza, ${ }^{84}$ M. Davier, ${ }^{22}$ G. S. Davies, ${ }^{33}$ E. J. Daw, ${ }^{85}$ R. Day, ${ }^{31}$ D. DeBra, ${ }^{38}$ G. Debreczeni, ${ }^{36}$ J. Degallaix, ${ }^{62}$ M. De Laurentis, ${ }^{64,4}$ S. Deléglise, ${ }^{58}$ W. Del Pozzo, ${ }^{43}$ T. Denker,${ }^{9,16}$ T. Dent, ${ }^{9}$ H. Dereli, ${ }^{51}$ V. Dergachev,${ }^{1}$ R. DeRosa,${ }^{6}$ R. De Rosa,${ }^{64,4}$ R. DeSalvo, ${ }^{8}$ S. Dhurandhar, ${ }^{13}$ M. C. Díaz,${ }^{84}$ L. Di Fiore, ${ }^{4}$ M. Di Giovanni, ${ }^{78,26}$ A. Di Lieto, ${ }^{17,18}$ I. Di Palma,${ }^{27,9}$ A. Di Virgilio, ${ }^{18}$ G. Dojcinoski, ${ }^{86}$ V. Dolique, ${ }^{62}$ F. Donovan, ${ }^{11}$ K. L. Dooley ${ }^{20}$ S. Doravari,${ }^{6}$ R. Douglas,${ }^{33}$ T. P. Downes, ${ }^{15}$ M. Drago,${ }^{9,87,88}$ R. W. P. Drever ${ }^{1}$ J. C. Driggers ${ }^{34}$ Z. Du, ${ }^{67}$ M. Ducrot,${ }^{7}$ S. E. Dwyer,${ }^{34}$ T. B. Edo, ${ }^{85}$ M. C. Edwards, ${ }^{76}$ A. Effler ${ }^{6}$ H.-B. Eggenstein, ${ }^{9}$ P. Ehrens, ${ }^{1}$ J. M. Eichholz, ${ }^{5}$ S. S. Eikenberry,${ }^{5}$ W. Engels, ${ }^{74}$ R. C. Essick,${ }^{11}$ T. Etzel, ${ }^{1}$ M. Evans, ${ }^{11}$ T. M. Evans, ${ }^{6}$ R. Everett, ${ }^{89}$ M. Factourovich, ${ }^{37}$ V. Fafone, ${ }^{69,70,77}$ H. Fair, ${ }^{32}$ S. Fairhurst, ${ }^{81}$ X. Fan, ${ }^{67}$ Q. Fang, ${ }^{49}$ S. Farinon, ${ }^{45}$ B. Farr,${ }^{73}$ W. M. Farr,${ }^{43}$ M. Favata, ${ }^{86}$ M. Fays,${ }^{81}$ H. Fehrmann, ${ }^{9}$ M. M. Fejer ${ }^{38}$ I. Ferrante, ${ }^{17,18}$ E. C. Ferreira, ${ }^{12}$ F. Ferrini, ${ }^{31}$ F. Fidecaro, ${ }^{17,18}$ I. Fiori ${ }^{31}$ R. P. Fisher, ${ }^{32}$ R. Flaminio, ${ }^{62}$ M. Fletcher, ${ }^{33}$ J.-D. Fournier, ${ }^{51}$ S. Franco,${ }^{22}$ S. Frasca, ${ }^{78,26}$ F. Frasconi ${ }^{18}$ Z. Frei, ${ }^{52}$ A. Freise, ${ }^{43}$ R. Frey, ${ }^{57}$ T. T. Fricke ${ }^{9}$ P. Fritschel, ${ }^{11}$ V. V. Frolov, ${ }^{6}$ P. Fulda, ${ }^{5}$ M. Fyffe, ${ }^{6}$ H. A. G. Gabbard,${ }^{20}$ J. R. Gair ${ }^{90}$ L. Gammaitoni, ${ }^{29,30}$ S. G. Gaonkar,${ }^{13}$ F. Garufi, ${ }^{64,4}$ A. Gatto, ${ }^{35}$ G. Gaur, ${ }^{91,92}$ N. Gehrels, ${ }^{65}$ G. Gemme,${ }^{45}$ B. Gendre, ${ }^{51}$ E. Genin, ${ }^{31}$ A. Gennai, ${ }^{18}$ J. George, ${ }^{46}$ L. Gergely, ${ }^{93}$ V. Germain, ${ }^{7}$ A. Ghosh, ${ }^{14}$ S. Ghosh,${ }^{10,50}$ J. A. Giaime,${ }^{2,6}$ K. D. Giardina,${ }^{6}$ A. Giazotto,${ }^{18}$ K. Gill, ${ }^{94}$ A. Glaefke, ${ }^{33}$ E. Goetz, ${ }^{68}$ R. Goetz, ${ }^{5}$ L. Gondan, ${ }^{52}$ 
G. González, ${ }^{2}$ J. M. Gonzalez Castro, ${ }^{17,18}$ A. Gopakumar, ${ }^{95}$ N. A. Gordon, ${ }^{33}$ M. L. Gorodetsky,${ }^{47}$ S. E. Gossan, ${ }^{1}$ M. Gosselin, ${ }^{31}$ R. Gouaty, ${ }^{7}$ C. Graef, ${ }^{33}$ P. B. Graff, ${ }^{65,60}$ M. Granata, ${ }^{62}$ A. Grant, ${ }^{33}$ S. Gras, ${ }^{11}$ C. Gray, ${ }^{34}$ G. Greco, ${ }^{55,56}$ A. C. Green, ${ }^{43}$ P. Groot, ${ }^{50}$ H. Grote, ${ }^{9}$ S. Grunewald,${ }^{27}$ G. M. Guidi, ${ }^{55,56}$ X. Guo ${ }^{67}$ A. Gupta, ${ }^{13}$ M. K. Gupta, ${ }^{92}$ K. E. Gushwa, ${ }^{1}$ E. K. Gustafson, ${ }^{1}$ R. Gustafson, ${ }^{68}$ J. J. Hacker, ${ }^{21}$ B. R. Hall, ${ }^{44}$ E. D. Hall, ${ }^{1}$ G. Hammond,${ }^{33}$ M. Haney, ${ }^{95}$ M. M. Hanke, ${ }^{9}$ J. Hanks, ${ }^{34}$ C. Hanna, ${ }^{89}$ M. D. Hannam, ${ }^{81}$ J. Hanson, ${ }^{6}$ T. Hardwick, ${ }^{2}$ J. Harms, ${ }^{55,56}$ G. M. Harry, ${ }^{96}$ I. W. Harry, ${ }^{27}$ M. J. Hart, ${ }^{33}$ M. T. Hartman, ${ }^{5}$ C.-J. Haster, ${ }^{43}$ K. Haughian, ${ }^{33}$ A. Heidmann, ${ }^{58}$ M. C. Heintze, ${ }^{5,6}$ H. Heitmann, ${ }^{51}$ P. Hello, ${ }^{22}$ G. Hemming, ${ }^{31}$ M. Hendry, ${ }^{33}$ I. S. Heng, ${ }^{33}$ J. Hennig, ${ }^{33}$ A. W. Heptonstall, ${ }^{1}$ M. Heurs,${ }^{9}{ }^{16}$ S. Hild,${ }^{33}$ D. Hoak, ${ }^{97}$ K. A. Hodge, ${ }^{1}$ D. Hofman, ${ }^{62}$ S. E. Hollitt,${ }^{98}$ K. Holt ${ }^{6}$ D. E. Holz, ${ }^{73}$ P. Hopkins, ${ }^{81}$ D. J. Hosken, ${ }^{98}$ J. Hough, ${ }^{33}$ E. A. Houston, ${ }^{33}$ E. J. Howell, ${ }^{49}$ Y. M. Hu, ${ }^{33}$ S. Huang, ${ }^{71}$ E. A. Huerta, ${ }^{99}$ D. Huet, ${ }^{22}$ B. Hughey, ${ }^{94}$ S. Husa, ${ }^{63}$ S. H. Huttner,${ }^{33}$ T. Huynh-Dinh, ${ }^{6}$ A. Idrisy, ${ }^{89}$ N. Indik, ${ }^{9}$ D. R. Ingram, ${ }^{34}$ R. Inta, ${ }^{80}$ H. N. Isa, ${ }^{33}$ J.-M. Isac, ${ }^{58}$ M. Isi, ${ }^{1}$ G. Islas, ${ }^{21}$ T. Isogai ${ }^{11}$ B. R. Iyer, ${ }^{14}$ K. Izumi, ${ }^{34}$ T. Jacqmin, ${ }^{58}$ H. Jang, ${ }^{75}$ K. Jani, ${ }^{61}$ P. Jaranowski, ${ }^{100}$ S. Jawahar, ${ }^{101}$ F. Jiménez-Forteza, ${ }^{63}$ W. W. Johnson, ${ }^{2}$ D. I. Jones, ${ }^{24}$ R. Jones, ${ }^{33}$ R.J.G. Jonker, ${ }^{10}$ L. Ju, ${ }^{49}$ Haris K, ${ }^{102}$ C. V. Kalaghatgi, ${ }^{23}$ V. Kalogera, ${ }^{103}$ S. Kandhasamy, ${ }^{20}$ G. Kang, ${ }^{75}$ J. B. Kanner, ${ }^{1}$ S. Karki, ${ }^{57}$ M. Kasprzack, ${ }^{2,22,31}$ E. Katsavounidis, ${ }^{11}$ W. Katzman, ${ }^{6}$ S. Kaufer, ${ }^{16}$ T. Kaur, ${ }^{49}$ K. Kawabe, ${ }^{34}$ F. Kawazoe, ${ }^{9}$ F. Kéfélian, ${ }^{51}$ M. S. Kehl, ${ }^{66}$ D. Keitel, ${ }^{9}$ D. B. Kelley, ${ }^{32}$ W. Kells, ${ }^{1}$ R. Kennedy, ${ }^{85}$ J. S. Key, ${ }^{84}$ A. Khalaidovski, ${ }^{9}$ F. Y. Khalili, ${ }^{47}$ S. Khan, ${ }^{81}$ Z. Khan, ${ }^{92}$ E. A. Khazanov, ${ }^{104}$ N. Kijbunchoo, ${ }^{34}$ C. Kim, ${ }^{75}$ J. Kim, ${ }^{105}$ K. Kim, ${ }^{106}$ N. Kim, ${ }^{75}$ N. Kim, ${ }^{38}$ Y.-M. Kim, ${ }^{105}$ E. J. King, ${ }^{98}$ P. J. King, ${ }^{34}$ D. L. Kinzel, ${ }^{6}$ J. S. Kissel, ${ }^{34}$ L. Kleybolte ${ }^{25}$ S. Klimenko, ${ }^{5}$ S. M. Koehlenbeck, ${ }^{9}$ K. Kokeyama, ${ }^{2}$ S. Koley, ${ }^{10}$ V. Kondrashov, ${ }^{1}$ A. Kontos, ${ }^{11}$ M. Korobko, ${ }^{25}$ W. Z. Korth, ${ }^{1}$ I. Kowalska, ${ }^{42}$ D. B. Kozak, ${ }^{1}$ V. Kringel, ${ }^{9}$ B. Krishnan, ${ }^{9}$ A. Królak, ${ }^{107,108}$ C. Krueger, ${ }^{16}$ G. Kuehn, ${ }^{9}$ P. Kumar, ${ }^{66}$ L. Kuo, ${ }^{71}$ A. Kutynia, ${ }^{107}$ B. D. Lackey, ${ }^{32}$ M. Landry, ${ }^{34}$ J. Lange, ${ }^{109}$ B. Lantz, ${ }^{38}$ P. D. Lasky, ${ }^{110}$ A. Lazzarini, ${ }^{1}$ C. Lazzaro, ${ }^{61,40}$ P. Leaci, ${ }^{27,78,26}$ S. Leavey, ${ }^{33}$ E. Lebigot,${ }^{35,67}$ C. H. Lee, ${ }^{105}$ H. K. Lee, ${ }^{106}$ H. M. Lee, ${ }^{111}$ K. Lee, ${ }^{33}$ A. Lenon, ${ }^{32}$ M. Leonardi, ${ }^{87,88}$ J. R. Leong, ${ }^{9}$ N. Leroy, ${ }^{22}$ N. Letendre, ${ }^{7}$ Y. Levin, ${ }^{110}$ B. M. Levine, ${ }^{34}$ T. G. F. Li,${ }^{1}$ A. Libson, ${ }^{11}$ T. B. Littenberg, ${ }^{103}$ N. A. Lockerbie, ${ }^{101}$ J. Logue, ${ }^{33}$ A. L. Lombardi, ${ }^{97}$ J. E. Lord, ${ }^{32}$ M. Lorenzini, ${ }^{77}$ V. Loriette, ${ }^{112}$ M. Lormand, ${ }^{6}$ G. Losurdo, ${ }^{56}$ J. D. Lough, ${ }^{9,16}$ H. Lück, ${ }^{16,9}$ A. P. Lundgren, ${ }^{9}$ J. Luo, ${ }^{76}$ R. Lynch, ${ }^{11}$ Y. Ma ${ }^{49}$ T. MacDonald, ${ }^{38}$ B. Machenschalk, ${ }^{9}$ M. MacInnis,${ }^{11}$ D. M. Macleod, ${ }^{2}$ F. Magaña-Sandoval, ${ }^{32}$ R. M. Magee, ${ }^{54}$ M. Mageswaran, ${ }^{1}$ E. Majorana, ${ }^{26}$ I. Maksimovic, ${ }^{112}$ V. Malvezzi, ${ }^{69,70}$ N. Man, ${ }^{51}$ I. Mandel, ${ }^{43}$ V. Mandic ${ }^{82}$ V. Mangano, ${ }^{78,26,33}$ G. L. Mansell, ${ }^{19}$ M. Manske, ${ }^{15}$ M. Mantovani, ${ }^{31}$ F. Marchesoni, ${ }^{113,30}$ F. Marion, ${ }^{7}$ S. Márka, ${ }^{37}$ Z. Márka, ${ }^{37}$ A. S. Markosyan, ${ }^{38}$ E. Maros,${ }^{1}$ F. Martelli, ${ }^{55,56}$ L. Martellini, ${ }^{51}$ I. W. Martin, ${ }^{33}$ R. M. Martin, ${ }^{5}$ D. V. Martynov ${ }^{1}$ J. N. Marx, ${ }^{1}$ K. Mason, ${ }^{11}$ A. Masserot,${ }^{7}$ T. J. Massinger ${ }^{32}$ M. Masso-Reid ${ }^{33}$ F. Matichard, ${ }^{11}$ L. Matone,${ }^{37}$ N. Mavalvala, ${ }^{11}$ N. Mazumder,${ }^{54}$ G. Mazzolo, ${ }^{9}$ R. McCarthy, ${ }^{34}$ D. E. McClelland, ${ }^{19}$ S. McCormick, ${ }^{6}$ S. C. McGuire, ${ }^{114}$ G. McIntyre, ${ }^{1}$ J. McIver,${ }^{97}$ D. J. McManus, ${ }^{19}$ S. T. McWilliams, ${ }^{99}$ D. Meacher,${ }^{51}$ G. D. Meadors,${ }^{27,9}$ J. Meidam, ${ }^{10}$ A. Melatos, ${ }^{83}$ G. Mendell, ${ }^{34}$ D. Mendoza-Gandara, ${ }^{9}$ R. A. Mercer, ${ }^{15}$ E. Merilh, ${ }^{34}$ M. Merzougui, ${ }^{51}$ S. Meshkov, ${ }^{1}$ C. Messenger ${ }^{33}$ C. Messick, ${ }^{89}$ P. M. Meyers ${ }^{82}$ F. Mezzani, ${ }^{26,78}$ H. Miao, ${ }^{43}$ C. Michel, ${ }^{62}$ H. Middleton, ${ }^{43}$ E. E. Mikhailov, ${ }^{115}$ L. Milano, ${ }^{64,4}$ J. Miller, ${ }^{11}$ M. Millhouse ${ }^{28}$ Y. Minenkov, ${ }^{70}$ J. Ming, ${ }^{27,9}$ S. Mirshekari, ${ }^{116}$ C. Mishra, ${ }^{14}$ S. Mitra,${ }^{13}$ V. P. Mitrofanov,${ }^{47}$ G. Mitselmakher, ${ }^{5}$ R. Mittleman, ${ }^{11}$ A. Moggi, ${ }^{18}$ M. Mohan, ${ }^{31}$ S. R. P. Mohapatra, ${ }^{11}$ M. Montani, ${ }^{55,56}$ B. C. Moore, ${ }^{86}$ C. J. Moore, ${ }^{90}$ D. Moraru, ${ }^{34}$ G. Moreno, ${ }^{34}$ S. R. Morriss, ${ }^{84}$ K. Mossavi, ${ }^{9}$ B. Mours, ${ }^{7}$ C. M. Mow-Lowry ${ }^{43}$ C. L. Mueller, ${ }^{5}$ G. Mueller, ${ }^{5}$ A. W. Muir ${ }^{81}$ Arunava Mukherjee, ${ }^{14}$ D. Mukherjee, ${ }^{15}$ S. Mukherjee,${ }^{84}$ A. Mullavey, ${ }^{6}$ J. Munch, ${ }^{98}$ D. J. Murphy, ${ }^{37}$ P. G. Murray, ${ }^{33}$ A. Mytidis, ${ }^{5}$ I. Nardecchia, ${ }^{69,70}$ L. Naticchioni, ${ }^{78,26}$ R. K. Nayak, ${ }^{117}$ V. Necula, ${ }^{5}$ K. Nedkova, ${ }^{97}$ G. Nelemans, ${ }^{10,50}$ M. Neri, ${ }^{44,45}$ A. Neunzert, ${ }^{68}$ G. Newton, ${ }^{33}$ T. T. Nguyen, ${ }^{19}$ A. B. Nielsen, ${ }^{9}$ S. Nissanke, ${ }^{50,10}$ A. Nitz, ${ }^{9}$ F. Nocera, ${ }^{31}$ D. Nolting, ${ }^{6}$ M. E. N. Normandin, ${ }^{84}$ L. K. Nuttall, ${ }^{32}$ J. Oberling, ${ }^{34}$ E. Ochsner, ${ }^{15}$ J. O'Dell, ${ }^{118}$ E. Oelker, ${ }^{11}$ G. H. Ogin, ${ }^{119}$ J. J. Oh, ${ }^{120}$ S. H. Oh, ${ }^{120}$ F. Ohme,${ }^{81}$ M. Oliver,${ }^{63}$ P. Oppermann, ${ }^{9}$ R. J. Oram, ${ }^{6}$ B. O'Reilly, ${ }^{6}$ R. O'Shaughnessy, ${ }^{109}$ C. D. Ott ${ }^{74}$ D. J. Ottaway, ${ }^{98}$ R. S. Ottens, ${ }^{5}$ 
H. Overmier, ${ }^{6}$ B. J. Owen, ${ }^{80}$ A. Pai, ${ }^{102}$ S. A. Pai, ${ }^{46}$ J. R. Palamos, ${ }^{57}$ O. Palashov, ${ }^{104}$ C. Palomba, ${ }^{26}$ A. Pal-Singh, ${ }^{25}$ H. Pan, ${ }^{71}$ C. Pankow, ${ }^{15,103}$ F. Pannarale, ${ }^{81}$ B. C. Pant,${ }^{46}$ F. Paoletti, ${ }^{31,18}$ A. Paoli, ${ }^{31}$ M. A. Papa, ${ }^{27,15,9}$ H. R. Paris, ${ }^{38}$ W. Parker, ${ }^{6}$ D. Pascucci, ${ }^{33}$ A. Pasqualetti, ${ }^{31}$ R. Passaquieti, ${ }^{17,18}$ D. Passuello, ${ }^{18}$ Z. Patrick, ${ }^{38}$ B. L. Pearlstone, ${ }^{33}$ M. Pedraza, ${ }^{1}$ R. Pedurand,${ }^{62}$ L. Pekowsky, ${ }^{32}$ A. Pele, ${ }^{6}$ S. Penn, ${ }^{121}$ R. Pereira,${ }^{37}$ A. Perreca, ${ }^{1}$ M. Phelps, ${ }^{33}$ O. Piccinni, ${ }^{78,26}$ M. Pichot,${ }^{51}$ F. Piergiovanni, ${ }^{55,56}$ V. Pierro, ${ }^{8}$ G. Pillant,${ }^{31}$ L. Pinard, ${ }^{62}$ I. M. Pinto, ${ }^{8}$ M. Pitkin, ${ }^{33}$ R. Poggiani, ${ }^{17,18}$ A. Post ${ }^{9}$ J. Powell, ${ }^{33}$ J. Prasad, ${ }^{13}$ V. Predoi, ${ }^{81}$ S. S. Premachandra, ${ }^{110}$ T. Prestegard, ${ }^{82}$ L. R. Price, ${ }^{1}$ M. Prijatelj, ${ }^{31}$ M. Principe, ${ }^{8}$ S. Privitera, ${ }^{27}$ G. A. Prodi,${ }^{87,88}$ L. Prokhorov, ${ }^{47}$ M. Punturo, ${ }^{30}$ P. Puppo, ${ }^{26}$ M. Pürrer, ${ }^{81}$ H. Qi ${ }^{15}$ J. Qin, ${ }^{49}$ V. Quetschke ${ }^{84}$ E. A. Quintero, ${ }^{1}$ R. QuitzowJames ${ }^{57}$ F. J. Raab, ${ }^{34}$ D. S. Rabeling, ${ }^{19}$ H. Radkins, ${ }^{34}$ P. Raffai,${ }^{52}$ S. Raja, ${ }^{46}$ M. Rakhmanov, ${ }^{84}$ P. Rapagnani, ${ }^{78,26}$ V. Raymond, ${ }^{27}$ M. Razzano, ${ }^{17,18}$ V. Re, ${ }^{69,70}$ J. Read, ${ }^{21}$ C. M. Reed, ${ }^{34}$ T. Regimbau, ${ }^{51}$ L. Rei, ${ }^{45}$ S. Reid, ${ }^{48}$ D. H. Reitze,${ }^{1,5}$ H. Rew,${ }^{115}$ F. Ricci, ${ }^{78,26}$ K. Riles, ${ }^{68}$ N. A. Robertson,,${ }^{1,33}$ R. Robie, ${ }^{33}$ F. Robinet, ${ }^{22}$ A. Rocchi, ${ }^{70}$ L. Rolland, ${ }^{7}$ J. G. Rollins, ${ }^{1}$ V. J. Roma, ${ }^{57}$ J. D. Romano, ${ }^{84}$ R. Romano, ${ }^{3,4}$ G. Romanov, ${ }^{115}$ J. H. Romie, ${ }^{6}$ D. Rosińska, ${ }^{122,41}$ S. Rowan, ${ }^{33}$ A. Rüdiger, ${ }^{9}$ P. Ruggi,${ }^{31}$ K. Ryan, ${ }^{34}$ S. Sachdev, ${ }^{1}$ T. Sadecki, ${ }^{34}$ L. Sadeghian, ${ }^{15}$ M. Saleem, ${ }^{102}$ F. Salemi, ${ }^{9}$ A. Samajdar, ${ }^{117}$ L. Sammut, ${ }^{83}$ E. J. Sanchez, ${ }^{1}$ V. Sandberg, ${ }^{34}$ B. Sandeen, ${ }^{103}$ J. R. Sanders,${ }^{68}$ B. Sassolas, ${ }^{62}$ B. S. Sathyaprakash, ${ }^{81}$ P. R. Saulson, ${ }^{32}$ O. Sauter ${ }^{68}$ R. L. Savage, ${ }^{34}$ A. Sawadsky, ${ }^{16}$ P. Schale, ${ }^{57}$ R. Schilling ${ }^{\dagger},{ }^{9}$ J. Schmidt,${ }^{9}$ P. Schmidt, ${ }^{1,74}$ R. Schnabel, ${ }^{25}$ R. M. S. Schofield,${ }^{57}$ A. Schönbeck, ${ }^{25}$ E. Schreiber, ${ }^{9}$ D. Schuette,${ }^{9,16}$ B. F. Schutz, ${ }^{81}$ J. Scott,${ }^{33}$ S. M. Scott,${ }^{19}$ D. Sellers, ${ }^{6}$ D. Sentenac,${ }^{31}$ V. Sequino, ${ }^{69,70}$ A. Sergeev, ${ }^{104}$ G. Serna, ${ }^{21}$ Y. Setyawati, ${ }^{50,10}$ A. Sevigny, ${ }^{34}$ D. A. Shaddock, ${ }^{19}$ S. Shah, ${ }^{10,50}$ M. S. Shahriar, ${ }^{103}$ M. Shaltev,${ }^{9}$ Z. Shao, ${ }^{1}$ B. Shapiro, ${ }^{38}$ P. Shawhan, ${ }^{60}$ A. Sheperd, ${ }^{15}$ D. H. Shoemaker, ${ }^{11}$ D. M. Shoemaker, ${ }^{61}$ K. Siellez, ${ }^{51}$ X. Siemens, ${ }^{15}$ D. Sigg, ${ }^{34}$ A. D. Silva, ${ }^{12}$ D. Simakov ${ }^{9}$ A. Singer, ${ }^{1}$ L. P. Singer, ${ }^{65}$ A. Singh, ${ }^{27,9}$ R. Singh ${ }^{2}$ A. M. Sintes, ${ }^{63}$ B. J. J. Slagmolen, ${ }^{19}$ J. R. Smith, ${ }^{21}$ N. D. Smith, ${ }^{1}$ R. J. E. Smith, ${ }^{1}$ E. J. Son, ${ }^{120}$ B. Sorazu, ${ }^{33}$ F. Sorrentino, ${ }^{45}$ T. Souradeep, ${ }^{13}$ A. K. Srivastava, ${ }^{92}$ A. Staley, ${ }^{37}$ M. Steinke, ${ }^{9}$ J. Steinlechner, ${ }^{33}$ S. Steinlechner, ${ }^{33}$ D. Steinmeyer, ${ }^{9,16}$ B. C. Stephens, ${ }^{15}$ R. Stone,${ }^{84}$ K. A. Strain, ${ }^{33}$ N. Straniero, ${ }^{62}$ G. Stratta, ${ }^{55,56}$ N. A. Strauss, ${ }^{76}$ S. Strigin, ${ }^{47}$ R. Sturani, ${ }^{116}$ A. L. Stuver ${ }^{6}$ T. Z. Summerscales, ${ }^{123}$ L. Sun, ${ }^{83}$ P. J. Sutton, ${ }^{81}$ B. L. Swinkels, ${ }^{31}$ M. J. Szczepanczyk, ${ }^{94}$ M. Tacca, ${ }^{35}$ D. Talukder, ${ }^{57}$ D. B. Tanner, ${ }^{5}$ M. Tápai, ${ }^{93}$ S. P. Tarabrin, ${ }^{9}$ A. Taracchini, ${ }^{27}$ R. Taylor,${ }^{1}$ T. Theeg, ${ }^{9}$ M. P. Thirugnanasambandam,${ }^{1}$ E. G. Thomas, ${ }^{43}$ M. Thomas,${ }^{6}$ P. Thomas,${ }^{34}$ K. A. Thorne, ${ }^{6}$ K. S. Thorne,${ }^{74}$ E. Thrane, ${ }^{110}$ S. Tiwari, ${ }^{77}$ V. Tiwari, ${ }^{81}$ K. V. Tokmakov, ${ }^{101}$ C. Tomlinson, ${ }^{85}$ M. Tonelli, ${ }^{17,18}$ C. V. Torres ${ }^{\ddagger},{ }^{84}$ C. I. Torrie,${ }^{1}$ D. Töyrä, ${ }^{43}$ F. Travasso, ${ }^{29,30}$ G. Traylor, ${ }^{6}$ D. Trifirò,${ }^{20}$ M. C. Tringali, ${ }^{87,88}$ L. Trozzo, ${ }^{124,18}$ M. Tse ${ }^{11}$ M. Turconi, ${ }^{51}$ D. Tuyenbayev, ${ }^{84}$ D. Ugolini, ${ }^{125}$ C. S. Unnikrishnan, ${ }^{95}$ A. L. Urban, ${ }^{15}$ S. A. Usman, ${ }^{32}$ H. Vahlbruch, ${ }^{16}$ G. Vajente, ${ }^{1}$ G. Valdes,${ }^{84}$ N. van Bakel,${ }^{10}$ M. van Beuzekom,${ }^{10}$ J. F. J. van den Brand, ${ }^{59,10}$ C. van den Broeck, ${ }^{10}$ D. C. Vander-Hyde, ${ }^{32,21}$ L. van der Schaaf,,${ }^{10}$ M. V. van der Sluys, ${ }^{10,50}$ J. V. van Heijningen, ${ }^{10}$ A. A. van Veggel, ${ }^{33}$ M. Vardaro, ${ }^{39,40}$ S. Vass,${ }^{1}$ M. Vasúth, ${ }^{36}$ R. Vaulin, ${ }^{11}$ A. Vecchio, ${ }^{43}$ G. Vedovato, ${ }^{40}$ J. Veitch, ${ }^{43}$ P. J. Veitch, ${ }^{98}$ K. Venkateswara, ${ }^{126}$ D. Verkindt, ${ }^{7}$ F. Vetrano, ${ }^{55,56}$ A. Viceré, ${ }^{55,56}$ S. Vinciguerra, ${ }^{43}$ D. J. Vine, ${ }^{48}$ J.Y. Vinet,${ }^{51}$ S. Vitale, ${ }^{11}$ T. Vo, ${ }^{32}$ H. Vocca, ${ }^{29,30}$ C. Vorvick, ${ }^{34}$ W. D. Vousden, ${ }^{43}$ S. P. Vyatchanin,${ }^{47}$ A. R. Wade, ${ }^{19}$ L. E. Wade ${ }^{15}$ M. Wade, ${ }^{15}$ M. Walker, ${ }^{2}$ L. Wallace, ${ }^{1}$ S. Walsh, ${ }^{15}$ G. Wang, ${ }^{77}$ H. Wang, ${ }^{43}$ M. Wang, ${ }^{43}$ X. Wang, ${ }^{67}$ Y. Wang, ${ }^{49}$ R. L. Ward, ${ }^{19}$ J. Warner,${ }^{34}$ M. Was, ${ }^{7}$ B. Weaver,${ }^{34}$ L.-W. Wei, ${ }^{51}$ M. Weinert, ${ }^{9}$ A. J. Weinstein, ${ }^{1}$ R. Weiss,${ }^{11}$ T. Welborn, ${ }^{6}$ L. Wen, ${ }^{49}$ P. Weßels, ${ }^{9}$ T. Westphal, ${ }^{9}$ K. Wette, ${ }^{9}$ J. T. Whelan, ${ }^{109,9}$ D. J. White, ${ }^{85}$ B. F. Whiting, ${ }^{5}$ R. D. Williams, ${ }^{1}$ A. R. Williamson, ${ }^{81}$ J. L. Willis, ${ }^{127}$ B. Willke, ${ }^{16,9}$ M. H. Wimmer, ${ }^{9,16}$ W. Winkler, ${ }^{9}$ C. C. Wipf,${ }^{1}$ H. Wittel, ${ }^{9,16}$ G. Woan, ${ }^{33}$ J. Worden, ${ }^{34}$ J. L. Wright,${ }^{33}$ G. Wu, ${ }^{6}$ J. Yablon, ${ }^{103}$ W. Yam, ${ }^{11}$ H. Yamamoto, ${ }^{1}$ C. C. Yancey, ${ }^{60}$ M. J. Yap, ${ }^{19}$ H. Yu, ${ }^{11}$ M. Yvert,${ }^{7}$ A. Zadrożny, ${ }^{107}$ L. Zangrando, ${ }^{40}$ M. Zanolin, ${ }^{94}$ J.-P. Zendri, ${ }^{40}$ M. Zevin, ${ }^{103}$ F. Zhang, ${ }^{11}$ L. Zhang, ${ }^{1}$ M. Zhang, ${ }^{115}$ Y. Zhang, ${ }^{109}$ C. Zhao, ${ }^{49}$ M. Zhou, ${ }^{103}$ Z. Zhou, ${ }^{103}$ X. J. Zhu, ${ }^{49}$ M. E. Zucker, ${ }^{11}$ S. E. Zuraw, ${ }^{97}$ and J. Zweizig ${ }^{1}$ ${ }^{\dagger}$ Deceased, May 2015. ${ }^{\ddagger}$ Deceased, March 2015. 


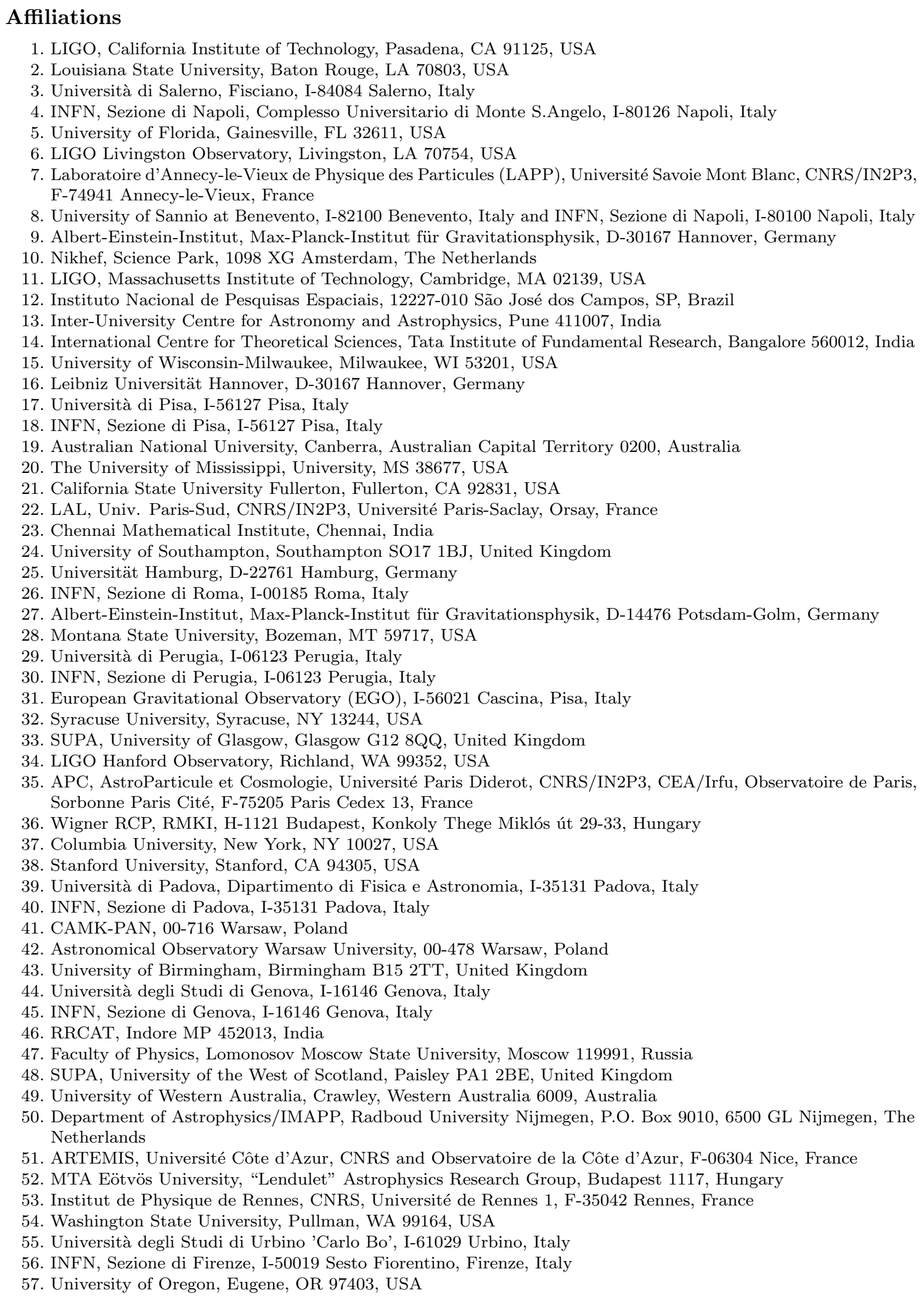


58. Laboratoire Kastler Brossel, UPMC-Sorbonne Universités, CNRS, ENS-PSL Research University, Collège de France, F-75005 Paris, France

59. VU University Amsterdam, $1081 \mathrm{HV}$ Amsterdam, The Netherlands

60. University of Maryland, College Park, MD 20742, USA

61. Center for Relativistic Astrophysics and School of Physics, Georgia Institute of Technology, Atlanta, GA 30332, USA

62. Laboratoire des Matériaux Avancés (LMA), IN2P3/CNRS, Université de Lyon, F-69622 Villeurbanne, Lyon, France

63. Universitat de les Illes Balears, IAC3-IEEC, E-07122 Palma de Mallorca, Spain

64. Università di Napoli 'Federico II', Complesso Universitario di Monte S.Angelo, I-80126 Napoli, Italy

65. NASA/Goddard Space Flight Center, Greenbelt, MD 20771, USA

66. Canadian Institute for Theoretical Astrophysics, University of Toronto, Toronto, Ontario M5S 3H8, Canada

67. Tsinghua University, Beijing 100084, China

68. University of Michigan, Ann Arbor, MI 48109, USA

69. Università di Roma Tor Vergata, I-00133 Roma, Italy

70. INFN, Sezione di Roma Tor Vergata, I-00133 Roma, Italy

71. National Tsing Hua University, Hsinchu City, Taiwan 30013, R.O.C.

72. Charles Sturt University, Wagga Wagga, New South Wales 2678, Australia

73. University of Chicago, Chicago, IL 60637, USA

74. Caltech CaRT, Pasadena, CA 91125, USA

75. Korea Institute of Science and Technology Information, Daejeon 305-806, Korea

76. Carleton College, Northfield, MN 55057, USA

77. INFN, Gran Sasso Science Institute, I-67100 L'Aquila, Italy

78. Università di Roma 'La Sapienza', I-00185 Roma, Italy

79. University of Brussels, Brussels 1050, Belgium

80. Texas Tech University, Lubbock, TX 79409, USA

81. Cardiff University, Cardiff CF24 3AA, United Kingdom

82. University of Minnesota, Minneapolis, MN 55455, USA

83. The University of Melbourne, Parkville, Victoria 3010, Australia

84. The University of Texas Rio Grande Valley, Brownsville, TX 78520, USA

85. The University of Sheffield, Sheffield S10 2TN, United Kingdom

86. Montclair State University, Montclair, NJ 07043, USA

87. Università di Trento, Dipartimento di Fisica, I-38123 Povo, Trento, Italy

88. INFN, Trento Institute for Fundamental Physics and Applications, I-38123 Povo, Trento, Italy

89. The Pennsylvania State University, University Park, PA 16802, USA

90. University of Cambridge, Cambridge CB2 1TN, United Kingdom

91. Indian Institute of Technology, Gandhinagar Ahmedabad Gujarat 382424, India

92. Institute for Plasma Research, Bhat, Gandhinagar 382428, India

93. University of Szeged, Dóm tér 9, Szeged 6720, Hungary

94. Embry-Riddle Aeronautical University, Prescott, AZ 86301, USA

95. Tata Institute for Fundamental Research, Mumbai 400005, India

96. American University, Washington, D.C. 20016, USA

97. University of Massachusetts-Amherst, Amherst, MA 01003, USA

98. University of Adelaide, Adelaide, South Australia 5005, Australia

99. West Virginia University, Morgantown, WV 26506, USA

100. University of Białystok, 15-424 Białystok, Poland

101. SUPA, University of Strathclyde, Glasgow G1 1XQ, United Kingdom

102. IISER-TVM, CET Campus, Trivandrum Kerala 695016, India

103. Northwestern University, Evanston, IL 60208, USA

104. Institute of Applied Physics, Nizhny Novgorod, 603950, Russia

105. Pusan National University, Busan 609-735, Korea

106. Hanyang University, Seoul 133-791, Korea

107. NCBJ, 05-400 Świerk-Otwock, Poland

108. IM-PAN, 00-956 Warsaw, Poland

109. Rochester Institute of Technology, Rochester, NY 14623, USA

110. Monash University, Victoria 3800, Australia

111. Seoul National University, Seoul 151-742, Korea

112. ESPCI, CNRS, F-75005 Paris, France

113. Università di Camerino, Dipartimento di Fisica, I-62032 Camerino, Italy

114. Southern University and A\&M College, Baton Rouge, LA 70813, USA

115. College of William and Mary, Williamsburg, VA 23187, USA

116. Instituto de Física Teórica, University Estadual Paulista/ICTP South American Institute for Fundamental Research, São Paulo SP 01140-070, Brazil 
117. IISER-Kolkata, Mohanpur, West Bengal 741252, India

118. Rutherford Appleton Laboratory, HSIC, Chilton, Didcot, Oxon OX11 0QX, United Kingdom

119. Whitman College, 280 Boyer Ave, Walla Walla, WA 9936, USA

120. National Institute for Mathematical Sciences, Daejeon 305-390, Korea

121. Hobart and William Smith Colleges, Geneva, NY 14456, USA

122. Institute of Astronomy, 65-265 Zielona Góra, Poland

123. Andrews University, Berrien Springs, MI 49104, USA

124. Università di Siena, I-53100 Siena, Italy

125. Trinity University, San Antonio, TX 78212, USA

126. University of Washington, Seattle, WA 98195, USA

127. Abilene Christian University, Abilene, TX 79699, USA 